\title{
Quantitative results of algebraic independence and Baker's method
}

\author{
by \\ Mamoru TAKEUCHI (Mito)
}

1. Introduction. It is well known that the method of A. Baker gives the transcendence of numbers related to exponential functions (see Theorems 2.3 and 2.4 of [2]). The idea of using Baker's method to obtain results of algebraic independence seems due to M. Waldschmidt [18], who makes a hypothesis on the transcendence type of the underlying field. In 1976, G. V. Chudnovsky announced some results of algebraic independence (transcendence degree 2), which was repeated in Chapter 2 of [5]. The complete proof of these last results can be seen in [8], where G. Diaz removed the complicated arguments coming from Kummer's theory by using the zero estimate of P. Philippon [13]. The result was also obtained by R. Tubbs [17] as a corollary to the general theorem on algebraic groups. G.-L. Chen [4] generalized Chudnovsky's result for exponential families to the case of large transcendence degree.

In this paper we shall establish quantitative results of this type in a general setting, by using Ably's method [1], which includes Chen's result. The improvement with respect to [1] comes from the construction of an auxiliary function with derivations. Furthermore if $\wp$ is a Weierstrass elliptic function with algebraic invariants, and if $\wp$ has no complex multiplications, we shall show that $\operatorname{deg} \operatorname{tr}_{\mathbb{Q}} \mathbb{Q}\left(\wp(u), \wp(\beta u), \wp\left(\beta^{2} u\right), \wp\left(\beta^{3} u\right)\right) \geq 2$, where $\beta$ is an algebraic number of degree 4 and $u$ is a complex number such that $u \notin \mathbb{Q}(\beta)$.

2. Notations and definitions. Let $G$ be a commutative algebraic group of dimension $d \geq 1$ defined over a number field $K$. Let $\mathbb{G}_{\mathrm{a}}$ denote the additive group of complex numbers and $\mathbb{G}_{\mathrm{m}}$ the multiplicative group of complex numbers. We suppose that $G$ decomposes as $G=\mathbb{G}_{\mathrm{a}}^{d_{0}} \times \mathbb{G}_{\mathrm{m}}^{d_{1}} \times G_{2}$, where $d_{0} \in\{0,1\}, d_{1} \geq 0$, and $G_{2}$ is a commutative algebraic group of dimension $d_{2}=d-d_{0}-d_{1}$, defined over $K$ and with no linear factor. The group $G_{2}(\mathbb{C})$ of complex points of $G_{2}$ is a complex Lie group. Let $\psi: \mathbb{C} \rightarrow G_{2}(\mathbb{C})$ be an an-

2000 Mathematics Subject Classification: Primary 11J85. 
alytic homomorphism whose tangent map at the origin Lie $\psi: \mathbb{C} \rightarrow T_{G_{2}}(\mathbb{C})$ is nontrivial, where $T_{G_{2}}(\mathbb{C})$ denotes the Lie algebra of $G_{2}$, identified with the tangent space at the origin, and let $\exp _{G_{2}}: T_{G_{2}}(\mathbb{C}) \rightarrow G_{2}(\mathbb{C})$ be its exponential map. We have $\psi=\exp _{G_{2}} \circ$ Lie $\psi$. Let $\chi_{2}: G_{2}(\mathbb{C}) \rightarrow \mathbb{P}_{N}(\mathbb{C})$ be the $K$-embedding of $G_{2}$ into projective $N$-space, as described by J.-P. Serre in [16]. Then $\chi_{2} \circ \exp _{G_{2}}: T_{G_{2}}(\mathbb{C}) \rightarrow \mathbb{P}_{N}(\mathbb{C})$ is given by analytic functions $\Theta_{0}, \ldots, \Theta_{N}$ (say) with order of growth at most 2 . Let $\chi$ be the natural $K$-embedding of $G(\mathbb{C})$ into $\mathbb{A}_{d_{0}}(\mathbb{C}) \times \mathbb{A}_{d_{1}}(\mathbb{C}) \times \mathbb{P}_{N}(\mathbb{C})$ associated to $\chi_{2}$, where $\mathbb{A}_{d}(\mathbb{C})$ denotes the set of complex points of the $d$-dimensional affine space $\mathbb{A}_{d}$.

Let $x_{1}, \ldots, x_{d_{1}}$ be complex numbers linearly independent over $\mathbb{Q}$, and $\varphi: \mathbb{C} \rightarrow G(\mathbb{C})$ the analytic homomorphism defined by

$$
\varphi(z)=\left(z, \exp \left(x_{1} z\right), \ldots, \exp \left(x_{d_{1}} z\right), \psi(z)\right) .
$$

In the definition of $\varphi$, if $d_{0}=0, d_{1}=0$ or $d_{2}=0$, we omit the corresponding component(s). Then we have $\varphi=\exp _{G} \circ \operatorname{Lie} \varphi$, where $\operatorname{Lie} \varphi$ is the tangent map of $\varphi$ at the origin.

For complex numbers $y_{1}, \ldots, y_{m}$ linearly independent over $\mathbb{Q}$, we put $Y=\mathbb{Z} y_{1}+\cdots+\mathbb{Z} y_{m}$ and $\Gamma=\varphi(Y)$. We put $\ell=\operatorname{rank}_{\mathbb{Z}}(Y \cap \operatorname{ker} \varphi)$ and we suppose $\ell<m$, hence we may assume without loss of generality that $y_{m-\ell+1}, \ldots, y_{m} \in \operatorname{ker} \varphi$. Let $L$ be an arbitrary subfield of $\mathbb{C}$. Let $W \subseteq T_{G}(\mathbb{C})$ denote a $\mathbb{C}$-vector subspace of $T_{G}(\mathbb{C})$ of least dimension which is defined over $L$ and which contains $\operatorname{Lie} \varphi(\mathbb{C})$. Put $n=\operatorname{dim}_{\mathbb{C}} W$, and suppose $n \geq d_{0}+1$. Let $\pi_{0}$ and $\pi_{1}$ be the projections of $G$ onto $\mathbb{G}_{\mathrm{a}}^{d_{0}}$ and $\mathbb{G}_{\mathrm{m}}^{d_{1}}$, respectively. Then we define the Dirichlet exponent $\mu^{\sharp}$ as in M. Waldschmidt [23] as follows:

$$
\mu^{\sharp}=\mu^{\sharp}(\Gamma, G, W)=\min _{G^{\prime} \subsetneq G} \frac{\eta+\delta_{1}+2 \delta_{2}}{\delta-\nu},
$$

where $G^{\prime}$ runs over all connected algebraic subgroups of $G$ which are defined over $K$, with $G^{\prime} \neq G$ and $\delta>\nu$, and where

$$
\begin{aligned}
& \eta=\operatorname{rank}_{\mathbb{Z}} \Gamma /\left(\Gamma \cap G^{\prime}\right), \quad \nu=\operatorname{dim}_{\mathbb{C}} W /\left(W \cap T_{G^{\prime}}(\mathbb{C})\right), \quad \delta=\operatorname{dim} G / G^{\prime}, \\
& \delta_{0}=\operatorname{dim} \mathbb{G}_{\mathrm{a}}^{d_{0}} / \pi_{0}\left(G^{\prime}\right), \quad \delta_{1}=\operatorname{dim} \mathbb{G}_{\mathrm{m}}^{d_{1}} / \pi_{1}\left(G^{\prime}\right), \quad \delta_{2}=\delta-\delta_{0}-\delta_{1} .
\end{aligned}
$$

We also define

$$
\kappa=\kappa\left(\mu^{\sharp}\right)=\frac{\mu^{\sharp}(d-n)-d_{1}-2 d_{2}}{(1-\ell / m) \mu^{\sharp}}+1 .
$$

Let $\underline{a}_{1}, \ldots, \underline{a}_{n}$ denote a fixed basis of $W$ over $\mathbb{C}$ such that all the components are in $L$, that is, $\underline{a}_{p}=\left(a_{1 p}, \ldots, a_{d p}\right), a_{h p} \in L(h=1, \ldots, d ; p=1, \ldots, n)$. By a linear transformation, we may suppose that $\Theta_{0}\left(\operatorname{Lie} \psi\left(y_{j}\right)\right) \neq 0$ for $1 \leq j \leq m-\ell$, and we put

$$
\begin{aligned}
& \underline{\omega}=\left(a_{h p}, y_{j}, \exp \left(x_{i} y_{j}\right)\right. \frac{\Theta_{s}\left(\operatorname{Lie} \psi\left(y_{j}\right)\right)}{\Theta_{0}\left(\operatorname{Lie} \psi\left(y_{j}\right)\right)} ; h=1, \ldots, d ; p=1, \ldots, n ; \\
&\left.i=1, \ldots, d_{1} ; j=1, \ldots, m-\ell ; s=0, \ldots, N\right),
\end{aligned}
$$


where as above, if $d_{0}=0, d_{1}=0$, or $d_{2}=0$, we omit the corresponding components.

Let $I$ be an ideal of $K\left[X_{1}, \ldots, X_{t}\right]$ and $\underline{\alpha}$ a point of $\mathbb{C}^{t}$. Recall the notions of the height and degree of $I$ and of the absolute value of $I$ at $\underline{\alpha}$, denoted by $\operatorname{Ht}(I), \operatorname{Deg}(I)$ and $\|I\|_{\underline{\alpha}}$, respectively, which were defined by P. Philippon in [12]; we define the size of $I$ by $T(I)=\log \operatorname{Ht}(I)+\operatorname{Deg}(I)$. For a polynomial $P \in K\left[X_{1}, \ldots, X_{t}\right]$, we define the size of $P$ by $t(P)=\max (1+\operatorname{deg} P, h(P))$, where $h(P)$ denotes the height of $P$ (see [12, définition 1.11]). For $\alpha \in \mathbb{C}^{t}$ and a positive real number $R$, we define $B^{t}(\underline{\alpha}, R)$ to be the open ball with center $\underline{\alpha}$ and radius $R$.

Definition. Let $\underline{\alpha} \in \mathbb{C}^{t}$. A function $\Phi: \mathbb{R}_{+} \rightarrow \mathbb{R}_{+}$is said to be a measure of algebraic independence of $\underline{\alpha}$ at dimension $k$ if for every ideal $J$ of $K\left[X_{1}, \ldots, X_{t}\right]$ of codimension $t-k$ and size $T(J)$ sufficiently large, we have

$$
\|J\|_{\underline{\alpha}} \geq \exp (-\Phi(T(J))) .
$$

For every algebraic subvariety $V$ of $\mathbb{P}=\mathbb{P}_{d_{0}} \times \mathbb{P}_{d_{1}} \times \mathbb{P}_{N}$ and real numbers $D_{0}, D_{1}, D_{2}>0$, we define $H\left(V ; D_{0}, D_{1}, D_{2}\right)$ as in [13] to be the homogeneous polynomial equal to $(\operatorname{dim} V)$ ! times the homogeneous part of (maximal) degree $(=\operatorname{dim} V)$ of the Hilbert-Samuel polynomial of $V$ evaluated at $\left(D_{0}, D_{1}, D_{2}\right)$.

Then we know from $\S 3$ of [13] that

$$
H\left(G ; D_{0}, D_{1}, D_{2}\right)=\frac{d ! \operatorname{deg} G_{2}}{d_{0} ! d_{1} ! d_{2} !} D_{0}^{d_{0}} D_{1}^{d_{1}} D_{2}^{d_{2}},
$$

and for a connected algebraic subgroup $G^{\prime}$ of $G$ with $G^{\prime} \neq G$,

$$
\begin{aligned}
& H\left(G^{\prime} ; D_{0}, D_{1}, D_{2}\right) \\
& \quad \geq \frac{(d-\delta) !}{\left(d_{0}-\delta_{0}\right) !\left(d_{1}-\delta_{1}\right) !\left(d_{2}-\delta_{2}\right) !} D_{0}^{d_{0}-\delta_{0}} D_{1}^{d_{1}-\delta_{1}} D_{2}^{d_{2}-\delta_{2}},
\end{aligned}
$$

where $\delta=\operatorname{dim} G / G^{\prime}, \delta_{0}=\operatorname{dim} \mathbb{G}_{\mathrm{a}}^{d_{0}} / \pi_{0}\left(G^{\prime}\right), \delta_{1}=\operatorname{dim} \mathbb{G}_{\mathrm{m}}^{d_{1}} / \pi_{1}\left(G^{\prime}\right)$, and $\delta_{2}=$ $\delta-\delta_{0}-\delta_{1}$.

In what follows, we denote by $c_{0}, c_{1}, c_{2}, \ldots$ real numbers depending only on $G,[K: \mathbb{Q}], \chi, x_{1}, \ldots, x_{d_{1}}, y_{1}, \ldots, y_{m}$, by $c$ a real number sufficiently large with respect to $c_{1}, c_{2}, \ldots$, and by $S_{0}$ a real number sufficiently large with respect to $c$.

For $\underline{t}=\left(t_{1}, \ldots, t_{m}\right) \in(\mathbb{N} \cup\{0\})^{m}$, we put $|\underline{t}|=t_{1}+\cdots+t_{m}$. Further for $\underline{h}=\left(h_{1}, \ldots, h_{m}\right) \in \mathbb{Z}^{m}$, we put $\|\underline{h}\|=\max _{1 \leq i \leq m}\left|h_{i}\right|$ and $\underline{h} \cdot \underline{y}=$ $h_{1} y_{1}+\cdots+h_{m} y_{m}$; and for real $S>0$, we put 


$$
\begin{aligned}
Y^{ \pm}(S) & =\left\{\underline{h} \cdot \underline{y} ;\left(h_{1}, \ldots, h_{m}\right) \in \mathbb{Z}^{m},\left|h_{i}\right|<S, 1 \leq i \leq m\right\}, \\
Y(S) & =\left\{\underline{h} \cdot \underline{y} ;\left(h_{1}, \ldots, h_{m}\right) \in \mathbb{Z}^{m}, 0 \leq h_{i}<S, 1 \leq i \leq m\right\}, \\
\Gamma(S) & =\varphi(Y(S)), \\
\mathbb{Z}^{m}(S) & =\left\{\underline{h} \in \mathbb{Z}^{m} ;\left|h_{i}\right|<S, 1 \leq i \leq m\right\}, \\
\mathbb{N}^{m}(S) & =\left\{\underline{h} \in \mathbb{N}^{m} ; 0 \leq h_{i}<S, 1 \leq i \leq m\right\} .
\end{aligned}
$$

As in [1], we put

$$
\begin{aligned}
A\left(S, G^{\prime}\right)=\left(\frac{1}{c}\left(S^{\mu^{\sharp}}(\log S)^{a-1}\right)^{\nu} \operatorname{card}\left(\left(\Gamma(S)+G^{\prime}\right) / G^{\prime}\right)\right. \\
\left.\quad \times \frac{H\left(G^{\prime} ; S^{\mu^{\sharp}}(\log S)^{-1}, S^{\mu^{\sharp}-1}, S^{\mu^{\sharp}-2}\right)}{H\left(G ; S^{\sharp}(\log S)^{-1}, S^{\mu^{\sharp}}-1, S^{\mu^{\sharp}-2}\right)}\right)^{1 / \delta}
\end{aligned}
$$

for $S \geq S_{0}$ and a connected algebraic subgroup $G^{\prime}$ of $G$ with $G^{\prime} \neq G$, where $a$ denotes a constant chosen below. It is clear that $\operatorname{card}\left(\left(\Gamma(S)+G^{\prime}\right) / G^{\prime}\right) \geq S^{\eta}$. We see from (1) and (2), and the definition of $\mu^{\sharp}$ that

$$
A\left(S, G^{\prime}\right) \geq\left(\frac{1}{c}\right)^{1 / \delta}(\log S)^{\left((a-1) \nu+\delta_{0}\right) / \delta}
$$

for all connected algebraic subgroups $G^{\prime} \subsetneq G$. We put

$$
A(S)=\min _{G^{\prime} \subsetneq G} A\left(S, G^{\prime}\right),
$$

where $G^{\prime}$ runs over all connected algebraic subgroups of $G$ with $G^{\prime} \neq G$, and we also put $B(S)=\min \left\{A(S), c^{-1}(\log S)^{\left((a-1) n+d_{0}\right) / d}\right\}$.

We introduce the following parameters:

$$
\begin{aligned}
& \bar{D}_{0}(S)= \begin{cases}S^{\mu^{\sharp}}(\log S)^{-1} B(S) & \text { if } d_{0} \neq 0, \\
1 & \text { otherwise, }\end{cases} \\
& \bar{D}_{1}(S)= \begin{cases}S^{\mu^{\sharp}-1} B(S) & \text { if } d_{1} \neq 0, \\
1 & \text { otherwise, }\end{cases} \\
& \bar{D}_{2}(S)= \begin{cases}S^{\mu^{\sharp}-2} B(S) & \text { if } d_{2} \neq 0, \\
1 & \text { otherwise, }\end{cases}
\end{aligned}
$$

and we put $D_{0}(S)=\left[\bar{D}_{0}(S)\right], D_{1}(S)=\left[\bar{D}_{1}(S)\right], D_{2}(S)=\left[\bar{D}_{2}(S)\right]$, where $[\xi]$ denotes the integral part of a real number $\xi$. Note that if $\mu^{\sharp}>i$ for $i=0,1,2$, then we have $D_{i}(S) \geq 1$ for all $S \geq S_{0}$.

As in [13], if $G$ is $K$-embedded in $\mathbb{P}=\mathbb{P}_{d_{0}} \times \mathbb{P}_{d_{1}} \times \mathbb{P}_{N}$, we say that a connected algebraic subgroup $G^{\prime}$ of $G$ is incompletely defined in $G$ by equations of multi-degree $\leq\left(D_{0}, D_{1}, D_{2}\right)$ if $G^{\prime}$ is an irreducible component of $G \cap Z(I)$, where $Z(I) \subset \mathbb{P}$ denotes the set of common zeros of an ideal $I$ of $K[\mathbb{P}]$ generated by polynomials of multi-degree $\leq\left(D_{0}, D_{1}, D_{2}\right)$. We fix a norm $\|\cdot\|$ on $T_{G}(\mathbb{C})$. 
We put

$$
S_{1}=c^{d+1}\left(S_{0}+1\right)^{m /(\kappa-1) \mu^{\sharp}}, \quad \Delta(S)=S^{\mu^{\sharp}}(\log S)^{a}, \quad \varrho(S)=S^{\kappa \mu^{\sharp}}(\log S)^{a},
$$

where $a$ is the same constant as above. Now we impose the following technical hypothesis which is similar to $(\mathrm{H})$ of [1]:

$\left(\mathrm{H}_{\mathrm{A}}\right) \quad$ There exist positive constants $c_{0}^{\prime}$ and $S_{0}$ such that for all $S \geq S_{0}$ and for all connected algebraic subgroups $G^{\prime}$ of $G$ with $G^{\prime} \subsetneq G \subset$ $\mathbb{P}_{d_{0}} \times \mathbb{P}_{d_{1}} \times \mathbb{P}_{d_{2}}$, incompletely defined in $G$ by equations of multidegree $\leq\left(\bar{D}_{0}(S), \bar{D}_{1}(S), 2 \bar{D}_{2}(S)\right)$, and for all $y \in Y^{ \pm}(S)$, we have

(i) if $y \neq 0$, then $|y| \geq \exp \left(-c_{0}^{\prime} S \log S\right)$,

(ii) either $\varphi(y) \in G^{\prime}(\mathbb{C})$ or for all $u \in T_{G}(\mathbb{C})$ such that $\exp _{G}(u) \in$ $G^{\prime}(\mathbb{C})$,

$$
\|u-\operatorname{Lie} \varphi(y)\| \geq \exp (-\varrho(S)) .
$$

Let $\theta_{1}, \ldots, \theta_{q}, \theta_{q+1}$ be complex numbers such that $\theta_{1}, \ldots, \theta_{q}$ are algebraic independent over $\mathbb{Q}, \theta_{q+1}$ integral over $\mathbb{Z}\left[\theta_{1}, \ldots, \theta_{q}\right]$ and $K(\underline{\omega})=$ $\mathbb{Q}\left(\theta_{1}, \ldots, \theta_{q+1}\right)$. Then the components of $\underline{\omega}$ can be written in the following forms:

$$
\begin{gathered}
a_{h p}=\frac{A_{h p}\left(\theta_{1}, \ldots, \theta_{q+1}\right)}{Q\left(\theta_{1}, \ldots, \theta_{q}\right)} \quad(h=1, \ldots, d ; p=1, \ldots, n), \\
y_{j}=\frac{B_{j}\left(\theta_{1}, \ldots, \theta_{q+1}\right)}{Q\left(\theta_{1}, \ldots, \theta_{q}\right)} \quad(j=1, \ldots, m-\ell), \\
e^{x_{i} y_{j}}=\frac{C_{i j}\left(\theta_{1}, \ldots, \theta_{q+1}\right)}{Q\left(\theta_{1}, \ldots, \theta_{q}\right)} \quad\left(i=1, \ldots, d_{1} ; j=1, \ldots, m-\ell\right), \\
\frac{\Theta_{s}\left(\operatorname{Lie} \psi\left(y_{j}\right)\right)}{\Theta_{0}\left(\operatorname{Lie} \psi\left(y_{j}\right)\right)}=\frac{D_{s j}\left(\theta_{1}, \ldots, \theta_{q+1}\right)}{Q\left(\theta_{1}, \ldots, \theta_{q}\right)} \quad(s=1, \ldots, N ; j=1, \ldots, m-\ell),
\end{gathered}
$$

where $A_{h p}, B_{j}, C_{i j}, D_{s j}$ and $Q$ are polynomials with coefficients in $\mathbb{Z}$.

Let $R\left(\theta_{1}, \ldots, \theta_{q}, X\right) \in \mathbb{Z}\left[\theta_{1}, \ldots, \theta_{q}\right][X]$ be the minimal polynomial of $\theta_{q+1}$ over $\mathbb{Z}\left[\theta_{1}, \ldots, \theta_{q}\right]$. Let $\underline{\tilde{\theta}}=\left(\widetilde{\theta}_{1}, \ldots, \widetilde{\theta}_{q}\right) \in B^{q}(\underline{\theta}, \exp (-c \varrho(S)))$. By using the semi-resultant of Chudnovsky, there exists a simple zero $\widetilde{\theta}_{q+1}$ of $R\left(\widetilde{\theta}_{1}, \ldots, \widetilde{\theta}_{q}, X\right)$ such that $\left|\widetilde{\theta}_{q+1}-\theta_{q+1}\right| \leq \exp (-(c / 2) \varrho(S))$ (cf. [21, p. 263]); and we use the same notation as above for the vector $\left(\widetilde{\theta}_{1}, \ldots, \widetilde{\theta}_{q+1}\right)$, i.e., $\underline{\tilde{\theta}}=\left(\widetilde{\theta}_{1}, \ldots, \widetilde{\theta}_{q+1}\right)$.

For any $\underline{\tilde{\theta}}=\left(\widetilde{\theta}_{1}, \ldots, \widetilde{\theta}_{q+1}\right)$ with $\underline{\tilde{\theta}} \in B^{q}(\underline{\theta}, \exp (-c \varrho(S)))$, let $\widetilde{a}_{h p}$ denote the fractions resulting when all $\theta_{i}$ in $a_{h p}$ are replaced by $\widetilde{\theta}_{i}(i=1, \ldots, q+1)$. Put $\widetilde{W}:=\mathbb{C}_{\widetilde{a}_{1}}+\cdots+\mathbb{C}_{n}$, where $\underline{\widetilde{a}}_{p}=\left(\widetilde{a}_{1 p}, \ldots, \widetilde{a}_{d p}\right)(p=1, \ldots, n)$. Now we shall impose the second hypothesis:

$\left(\mathrm{H}_{\mathrm{B}}\right) \quad$ For all $S \geq S_{1}$, for all $\underline{\tilde{\theta}}=\left(\widetilde{\theta}_{1}, \ldots, \widetilde{\theta}_{q}\right) \in B^{q}(\underline{\theta}, \exp (-c \varrho(S)))$ and for all connected algebraic subgroups $G^{\prime}$ of $G$ with $G^{\prime} \neq G$, incom- 
pletely defined by equations of multi-degree $\leq\left(\bar{D}_{0}, \bar{D}_{1}, 2 \bar{D}_{2}\right)$, the following inequality holds:

$$
\operatorname{codim}_{\widetilde{W}}\left(\widetilde{W} \cap T_{G^{\prime}}(\mathbb{C})\right) \geq \operatorname{codim}_{W}\left(W \cap T_{G^{\prime}}(\mathbb{C})\right) .
$$

3. The main result and corollaries. We shall prove the following theorem.

Theorem. Suppose that hypotheses $\left(\mathrm{H}_{\mathrm{A}}\right)$ and $\left(\mathrm{H}_{\mathrm{B}}\right)$ are satisfied and that $\kappa>1$, and if $G$ is nonlinear, then also $\mu^{\sharp}>2$. Let $k$ be an integer $\geq 0$ such that $\kappa \geq k+1$. Then there exists a real number

$$
c_{1}=c_{1}\left(G, \chi, \varphi,[K: \mathbb{Q}], L, x_{1}, \ldots, x_{d_{1}}, y_{1}, \ldots, y_{m}, k\right)>0
$$

such that

(i) if $\kappa=k+1$, the function

$$
\Phi_{1}(T)=\exp \left(c_{1} T^{\frac{d-n}{(\kappa-1)\left(n-d_{0}\right)}}\right)
$$

is a measure of algebraic independence of $\underline{\omega}$ at dimension $k$,

(ii) if $\kappa>k+1$, the function

$$
\Phi_{2}(T)=c_{1}\left(\frac{T}{(\log T)^{\frac{(k+1)(\kappa-1)\left(n-d_{0}\right)}{(d-n) \kappa}}}\right)^{\kappa /(\kappa-k-1)}
$$

is a measure of algebraic independence of $\underline{\omega}$ at dimension $k$.

CoROLlary 1. Under the assumptions of the Theorem, we have

$$
\operatorname{deg} \operatorname{tr}_{\mathbb{Q}} K(\underline{\omega}) \geq[\kappa] .
$$

REMARK. We shall compare our result with Ably's in the special case that the Dirichlet exponent $\mu^{\sharp}$ attains its minimum when $G^{\prime}=\{0\}$, where $G^{\prime}$ is a connected algebraic subgroup of $G$ with $G^{\prime} \neq G$, and furthermore we suppose $\ell=0$, since otherwise this is complicated. To avoid confusion, we shall denote the quantities $\mu^{\sharp}$ and $\kappa$ by $\mu^{\sharp}(A)$ and $\kappa(A)$ in Ably's case, and by $\mu^{\sharp}(T)$ and $\kappa(T)$ in our case. Then under the above assumption, we have

$$
\begin{array}{ll}
\mu^{\sharp}(A)=\left(m+d_{1}+2 d_{2}\right) / d, & \kappa(A)=d m /\left(m+d_{1}+2 d_{2}\right), \\
\mu^{\sharp}(T)=\left(m+d_{1}+2 d_{2}\right) /(d-n), & \kappa(T)=1+(d-n) m /\left(m+d_{1}+2 d_{2}\right) .
\end{array}
$$

Hence if $[\kappa(T)]>[\kappa(A)]$, our result is better than Ably's, and otherwise the latter is better. However, one must bear in mind that our result requires some superfluous assumptions.

Now we shall state some corollaries derived from our Theorem. Let $z_{1}, \ldots, z_{t}$ be complex numbers and $L$ an arbitrary subfield of $\mathbb{C}$. Let $s$ denote the number of elements among $\left\{z_{1}, \ldots, z_{t}\right\}$ linearly independent over $L$. For 
simplicity, we shall assume that $z_{1}, \ldots, z_{s}$ (say) are linearly independent over $L$, and so we can write $z_{h}=\sum_{p=1}^{s} a_{h p} z_{p}, a_{h p} \in L(h=1, \ldots, t)$.

Then we shall need the following definition similar to that of Chen [4].

Definition. Let $\alpha$ be a positive number and $\tau$ an irrational number. We say that a family $\left\{z_{1}, \ldots, z_{t}\right\}$ of complex numbers satisfies hypothesis $\mathrm{H}(L, \mathbb{Z} ; \alpha)$ (resp. $\mathrm{H}(L, \mathbb{Z}+\tau \mathbb{Z} ; \alpha))$ if there exist positive constants $c_{*}$ and $N_{*}$ such that for all integers $N \geq N_{*}$, all integers $k$ with $1 \leq k \leq s$ and all $\lambda_{i_{1} \ldots i_{k}} \in \mathbb{Z}\left(\right.$ resp. $\left.\lambda_{i_{1}, \ldots, i_{k}} \in \mathbb{Z}+\tau \mathbb{Z}\right)\left(1 \leq i_{1}<\cdots<i_{k} \leq t\right)$ satisfying $\left|\lambda_{i_{1} \ldots i_{k}}\right| \leq N$, we have either

or

$$
\max _{1 \leq j_{1}<\cdots<j_{k} \leq s}\left|\sum_{1 \leq i_{1}<\cdots<i_{k} \leq t} \lambda_{i_{1} \ldots i_{k}} \operatorname{det}\left(a_{i_{u} j_{v}}\right)_{1 \leq u, v \leq k}\right|=0,
$$

$$
\max _{1 \leq j_{1}<\cdots<j_{k} \leq s}\left|\sum_{1 \leq i_{1}<\cdots<i_{k} \leq t} \lambda_{i_{1} \ldots i_{k}} \operatorname{det}\left(a_{i_{u} j_{v}}\right)_{1 \leq u, v \leq k}\right| \geq \exp \left(-c_{*} N^{\alpha}\right) .
$$

Algebraic independence of values of the exponential function. Let $x_{1}, \ldots$ $\ldots, x_{d_{1}}$ (resp. $\left.y_{1}, \ldots, y_{m}\right)$ be $\mathbb{Q}$-linearly independent complex numbers. Let $L$ be an arbitrary subfield of $\mathbb{C}$. Let $r$ (resp. $r+1$ ) denote the number of elements among $\left\{x_{1}, \ldots, x_{d_{1}}\right\}$ (resp. $\left\{1, x_{1}, \ldots, x_{d_{1}}\right\}$ ) linearly independent over $L$. As above, for brevity, we shall assume that $\left\{x_{1}, \ldots, x_{r}\right\}$ (resp. $\left.\left\{1, x_{1}, \ldots, x_{r}\right\}\right)$ are linearly independent over $L$, and hence $x_{h}=\sum_{p=1}^{r} a_{h p} x_{p}$ $\left(h=1, \ldots, d_{1}\right)\left(\right.$ resp. $\left.x_{h}=\sum_{p=0}^{r} a_{h p} x_{p}\left(h=0, \ldots, d_{1}\right), x_{0}:=1\right)$. Put

$$
\begin{aligned}
\kappa_{1} & =\left(d_{1}-r\right) m /\left(m+d_{1}\right)+1, \quad \mu_{1}^{\sharp}=\left(m+d_{1}\right) /\left(d_{1}-r\right), \quad \varrho_{1}=\left(m+d_{1}\right) / m r, \\
\underline{\omega}^{(1)} & =\left(a_{h p}, e^{x_{i} y_{j}} ; h=1, \ldots, d_{1}, p=1, \ldots, r, i=1, \ldots, d_{1}, j=1, \ldots, m\right), \\
\underline{\omega}^{(2)} & =\left(a_{h p}, y_{j}, e^{x_{i} y_{j}} ; h=0, \ldots, d_{1}, p=0, \ldots, r, i=1, \ldots, d_{1}, j=1, \ldots, m\right) .
\end{aligned}
$$

We consider the following technical hypothesis.

$\left(\mathrm{H}_{1}\right) \quad$ There exist $c_{1}^{\prime}, S_{1}^{\prime}>0$ such that for all $S \geq S_{1}^{\prime}$ and for all $\underline{\lambda}=$ $\left(\lambda_{1}, \ldots, \lambda_{d_{1}}\right)$ not all zero in $\mathbb{Z}^{n}$ satisfying $\|\underline{\lambda}\| \leq S$ (resp. for all $\underline{h}=\left(h_{1}, \ldots, h_{m}\right)$ not all zero in $\mathbb{Z}^{m}$ satisfying $\left.\|\underline{h}\| \leq S\right)$, we have

resp.

$$
\left|\sum_{i=1}^{d_{1}} \lambda_{i} x_{i}\right| \geq \exp \left(-S^{\left(\mu_{1}^{\sharp}+m\right) /\left(2 \mu_{1}^{\sharp}-1\right)}\right),
$$

$$
\left|\sum_{j=1}^{m} h_{j} y_{j}\right| \geq \max \left(\exp \left(-c_{1}^{\prime} S \log S\right), \exp \left(-S^{\left(\mu_{1}^{\sharp}+m\right) /\left(\mu_{1}^{\sharp}+1\right)}\right)\right) .
$$

COROllary 2. Let $\sigma=1$ or 2. Suppose that $\kappa_{1}>1$ and that hypotheses $\left(\mathrm{H}_{1}\right)$ and $\mathrm{H}\left(L, \mathbb{Z} ;\left(\mu_{1}^{\sharp}+m\right) / d_{1}\left(\mu_{1}^{\sharp}-1\right)\right)$ hold. Let $k$ be an integer such that $\kappa_{1} \geq$ $k+1$. Then there exists a real number $c_{1}=c_{1}\left(x_{1}, \ldots, x_{d_{1}}, y_{1}, \ldots, y_{m}, L, k\right)$ $>0$ such that 
(i) if $\kappa_{1}=k+1$, the function $\Phi_{1}(T)=\exp \left(c_{1} T^{\varrho_{1}}\right)$ is a measure of algebraic independence of $\underline{\omega}^{(\sigma)}$ at dimension $k$,

(ii) if $\kappa_{1}>k+1$, the function $\Phi_{2}(T)=c_{1}\left(T /(\log T)^{(k+1) / \kappa_{1} \varrho_{1}}\right)^{\kappa_{1} /\left(\kappa_{1}-k-1\right)}$ is a measure of algebraic independence of $\underline{\omega}^{(\sigma)}$ at dimension $k$. have

Corollary 3 (see Chen [4]). Under the assumptions of Corollary 2, we

$$
\operatorname{deg} \operatorname{tr}_{\mathbb{Q}} \mathbb{Q}\left(\underline{\omega}^{(\sigma)}\right) \geq\left[\kappa_{1}\right] \quad(\sigma=1,2) .
$$

Corollary 4. Let $\alpha \neq 0,1$ and $\beta$ be algebraic numbers with $\operatorname{deg}(\beta)=5$. Then

$$
\operatorname{deg} \operatorname{tr}_{\mathbb{Q}} \mathbb{Q}\left(\alpha^{\beta}, \alpha^{\beta^{2}}, \alpha^{\beta^{3}}, \alpha^{\beta^{4}}\right) \geq 3
$$

Algebraic independence of values of a Weierstrass elliptic function. We shall deduce from our Theorem the elliptic analogue of the preceding results. Let $\wp$ be a Weierstrass elliptic function with algebraic invariants $g_{2}$ and $g_{3}$, $\Omega$ the lattice of periods, and $\omega_{1}, \omega_{2}$ a fixed basis for $\Omega$. We put $\tau=\omega_{2} / \omega_{1}$. Let $\mathbb{F}$ be the field of multiplications of $\wp$, and $O(\mathbb{F})$ the ring of integers of $\mathbb{F}$. Let $x_{1}, \ldots, x_{d_{1}}$ (resp. $y_{1}, \ldots, y_{m}$ ) be $\mathbb{F}$-linearly independent complex numbers. We suppose that $2 m \leq m d_{1}-1$ if $\wp$ has complex multiplications, and $2 m \leq m d_{1}+d_{1}-3$ otherwise. Let $L$ be an arbitrary subfield of $\mathbb{C}$. Let $r$ (resp. $r+1$ ) denote the number of elements among $\left\{x_{1}, \ldots, x_{d_{1}}\right\}$ (resp. $\left\{1, x_{1}, \ldots, x_{d_{1}}\right\}$ ) linearly independent over $L$, and let $a_{h p}$ be as in the exponential case. We put

$$
\begin{aligned}
& \kappa_{2}=[\mathbb{F}: \mathbb{Q}]\left(d_{1}-r\right) m /\left([\mathbb{F}: \mathbb{Q}] m+2 d_{1}\right)+1, \quad \mu_{2}^{\sharp}=\left([\mathbb{F}: \mathbb{Q}] m+2 d_{1}\right) /\left(d_{1}-r\right), \\
& \varrho_{2}=\left([\mathbb{F}: \mathbb{Q}] m+2 d_{1}\right) / r m, \\
& \underline{\omega}^{(3)}=\left(a_{h p}, \wp\left(x_{i} y_{j}\right) ; h=1, \ldots, d_{1}, p=1, \ldots, r, i=1, \ldots, d_{1}, j=1, \ldots, m,\right.\left.x_{i} y_{j} \notin \Omega\right), \\
& \underline{\omega}^{(4)}=\left(a_{h p}, y_{j}, \wp\left(x_{i} y_{j}\right) ; h=0, \ldots, d_{1}, p=0, \ldots, r, i=1, \ldots, d_{1},\right. \\
&\left.j=1, \ldots, m, x_{i} y_{j} \notin \Omega\right) .
\end{aligned}
$$

We consider the following technical hypothesis.

$\left(\mathrm{H}_{2}\right) \quad$ There exist $c_{2}^{\prime}, S_{2}^{\prime}>0$ such that for all $S \geq S_{2}^{\prime}$ and for all $\underline{\lambda}=$ $\left(\lambda_{1}, \ldots, \lambda_{d_{1}}\right)$ not all zero in $(O(\mathbb{F}))^{d_{1}}$ satisfying $\|\underline{\lambda}\| \leq S$ (resp. for all $\underline{h}=\left(h_{1}, \ldots, h_{m}\right)$ not all zero in $(O(\mathbb{F}))^{m}$ satisfying $\left.\|\underline{h}\| \leq S\right)$, we have

$$
\left|\sum_{i=1}^{d_{1}} \lambda_{i} x_{i}\right| \geq \exp \left(-S^{\left([\mathbb{F}: \mathbb{Q}] m+\mu_{2}^{\sharp}\right) / 18 \mu_{2}^{\sharp}}\right),
$$

resp.

$$
\left|\sum_{j=1}^{m} h_{j} y_{j}\right| \geq \max \left(\exp \left(-c_{2}^{\prime} S \log S\right), \exp \left(-S^{\left([\mathbb{F}: \mathbb{Q}] m+\mu_{2}^{\sharp}\right) / 4 \mu_{2}^{\sharp}}\right)\right) .
$$


COROllary 5. Let $\sigma=3$ or 4 . Suppose that $\kappa_{2}>1$ and that hypotheses $\left(\mathrm{H}_{2}\right)$ and $\mathrm{H}\left(L, \mathbb{Z}+\mathbb{Z} \tau ;\left([\mathbb{F}: \mathbb{Q}] m+\mu_{2}^{\sharp}\right) / 2 d_{1}\left(1+\log d_{1}\right)\left(\mu_{2}^{\sharp}-2\right)\right)$ are satisfied. Let $k$ be an integer such that $\kappa_{2} \geq k+1$. Then there exists a real number $c_{1}=c_{1}\left(x_{1}, \ldots, x_{d_{1}}, y_{1}, \ldots, y_{m}, k, L\right)>0$ such that

(i) if $\kappa_{2}=k+1$, the function $\Phi_{1}(T)=\exp \left(c_{1} T^{\varrho_{2}}\right)$ is a measure of algebraic independence of $\underline{\omega}^{(\sigma)}$ at dimension $k$,

(ii) if $\kappa_{2}>k+1$, the function $\Phi_{2}(T)=c_{1}\left(T /(\log T)^{(k+1) / \kappa_{2} \varrho_{2}}\right)^{\kappa_{2} /\left(\kappa_{2}-k-1\right)}$ is a measure of algebraic independence of $\underline{\omega}^{(\sigma)}$ at dimension $k$.

COROLlary 6. Under the assumptions of Corollary 5, we have

$$
\operatorname{deg} \operatorname{tr}_{\mathbb{Q}} \mathbb{Q}\left(\underline{\omega}^{(\sigma)}\right) \geq\left[\kappa_{2}\right] \quad(\sigma=3,4) .
$$

COROLlary 7. Let $\wp$ be a Weierstrass elliptic function with algebraic invariants. Let $E$ be the elliptic curve associated to $\wp$. Let $\beta$ be an algebraic number of degree $\delta \geq 2$ over $\mathbb{F}$ and $u$ a complex number such that $\wp(u), \wp(\beta u), \ldots, \wp\left(\beta^{\delta-1} u\right)$ are defined and $u \notin \mathbb{Q}(\beta)$. Suppose that $\delta>2 /[\mathbb{F}: \mathbb{Q}]$. Then:

(i) if $\wp$ has no complex multiplications $(\mathbb{F}=\mathbb{Q})$ and $\delta>2$, we have

$$
\operatorname{deg} \operatorname{tr}_{\mathbb{Q}} \mathbb{Q}\left(\wp(u), \wp(\beta u), \ldots, \wp\left(\beta^{\delta-1} u\right)\right) \geq\left[\frac{\delta+2}{3}\right],
$$

(ii) if $\wp$ has complex multiplications $([\mathbb{F}: \mathbb{Q}]=2)$ and $\delta \geq 2$, we have

$$
\operatorname{deg} \operatorname{tr}_{\mathbb{Q}} \mathbb{Q}\left(\wp(u), \wp(\beta u), \ldots, \wp\left(\beta^{\delta-1} u\right)\right) \geq\left[\frac{\delta+1}{2}\right] .
$$

REMARK. In Corollary 7 , if $\wp$ has no complex multiplications and $\operatorname{deg}(\beta)$ $=4$, we have $\operatorname{deg} \operatorname{tr}_{\mathbb{Q}} \mathbb{Q}\left(\wp(u), \wp(\beta u), \wp\left(\beta^{2} u\right), \wp\left(\beta^{3} u\right)\right) \geq 2$.

4. Propositions. We shall use the notations of $\S 2$. For every $j$ with $1 \leq$ $j \leq m-\ell$, a point $\gamma_{j}=\varphi\left(y_{j}\right)=\exp _{G}\left(\operatorname{Lie} \varphi\left(y_{j}\right)\right)$ has projective coordinates

$$
\left(Q(\underline{\theta}), B_{j}(\underline{\theta}), Q(\underline{\theta}), C_{1 j}(\underline{\theta}), \ldots, C_{d_{1}, j}(\underline{\theta}), Q(\underline{\theta}), D_{1 j}(\underline{\theta}), \ldots, D_{N j}(\underline{\theta})\right)
$$

in $\mathbb{P}_{d_{0}}(\mathbb{C}) \times \mathbb{P}_{d_{1}}(\mathbb{C}) \times \mathbb{P}_{N}(\mathbb{C})$. For every $j$ with $1 \leq j \leq m-\ell$, we let $\widetilde{\gamma}_{j}$ be the point with multiprojective coordinates given by evaluating the coordinate polynomials of $(3)$ at $\underline{\widetilde{\theta}}$. Then $\widetilde{\gamma}_{j} \in G(\mathbb{C})$ (cf. [21, §5]). Further, for $j=1, \ldots, m-\ell$, there exists $\widetilde{y}_{j} \in T_{G}(\mathbb{C})$ such that $\exp _{G}\left(\widetilde{y}_{j}\right)=\widetilde{\gamma}_{j}$ and $\left\|\operatorname{Lie} \varphi\left(y_{j}\right)-\widetilde{y}_{j}\right\| \leq \exp (-(c / 2) \varrho(S))$, since $\exp _{G}$ is a local diffeomorphism. Put $\widetilde{Y}=\mathbb{Z} \widetilde{y}_{1}+\cdots+\mathbb{Z} \widetilde{y}_{m-\ell}, \widetilde{\Gamma}=\exp _{G}(\widetilde{Y})$; and for $\underline{h}=\left(h_{1}, \ldots, h_{m}\right) \in \mathbb{Z}^{m}$, put $\underline{h} \cdot \underline{y}=h_{1} y_{1}+\cdots+h_{m} y_{m} \in \mathbb{C}$ and $\underline{h} \cdot \widetilde{y}=h_{1} \widetilde{y}_{1}+\cdots+h_{m-\ell} \widetilde{y}_{m-\ell} \in T_{G}(\mathbb{C})$.

We identify $T_{G}(\mathbb{C})$ with $\mathbb{C}^{d_{0}} \oplus \mathbb{C}^{d_{1}} \oplus \mathbb{C}^{d_{2}}$, and for $i=0,1,2$ we denote by $p_{i}$ the projection of $T_{G}(\mathbb{C})$ onto $\mathbb{C}^{d_{i}}$; hence $\widetilde{y} \in T_{G}(\mathbb{C})$ can be written as $\widetilde{y}=p_{0}(\widetilde{y})+p_{1}(\widetilde{y})+p_{2}(\widetilde{y})$.

We use the following criterion for algebraic independence: 
Proposition A ([1, p. 207]). Let $\underline{\alpha}=\left(\alpha_{1}, \ldots, \alpha_{t}\right) \in \mathbb{C}^{t}, k \in\{0, \ldots, t-1\}$, and $K$ a number field. Let $u: \mathbb{R}_{+} \rightarrow \mathbb{R}_{+}$be a continuous and strictly increasing function. Suppose that there exist $c_{0} \geq 1$ and $N_{0}>0$ such that for every real $N \geq N_{0}$, there exists an ideal $I_{N}=\left(G_{N, 1}, \ldots, G_{N, m(N)}\right)$ of $K\left[X_{1}, \ldots, X_{t}\right]$ satisfying

(i) the set of zeros of $I_{N}$ in $B^{t}\left(\underline{\alpha}, \exp \left(-c_{0} N^{k+1} u(N)\right)\right)$ is empty,

(ii) $\max _{1 \leq j \leq m(N)}\left|G_{N, j}(\underline{\alpha})\right| \leq \exp \left(-N^{k+1} u(N)\right)$,

(iii) $\max _{1 \leq j \leq m(N)} t\left(G_{N, j}\right) \leq N$.

Then if $v$ denotes the inverse function of $u$, there exists $c_{2}=c_{2}\left(c_{0}, t, k\right.$, $[K: \mathbb{Q}])>0$ such that the function $\Phi(T)=c_{2} T\left(v\left(c_{2} T\right)\right)^{k+1}$ is a measure of algebraic independence of $\underline{\alpha}$ at dimension $k$.

The proof of our Theorem will be established by combining the following result with Proposition A. Recall that the constant $c$ occurred in the definition of $A\left(S, G^{\prime}\right)$.

Proposition B. Suppose that hypotheses $\left(\mathrm{H}_{\mathrm{A}}\right)$ and $\left(\mathrm{H}_{\mathrm{B}}\right)$ are satisfied and that $\kappa>1$, and $\mu^{\sharp}>2$ if $G$ is nonlinear. Then for all $S \geq S_{1}$ there exists an ideal $\mathcal{I}_{S}=\left(P_{S, 1}, \ldots, P_{S, m(S)}\right)$ in $K\left[X_{1}, \ldots, X_{q}\right]$ such that

(i) the set of zeros of $\mathcal{I}_{S}$ in $B^{q}(\underline{\theta}, \exp (-c \varrho(S)))$ is empty,

(ii) $\max _{1 \leq i \leq m(S)}\left|P_{S, i}(\underline{\theta})\right| \leq \exp \left(-c_{3} \varrho(S)\right)$,

(iii) $\max _{1 \leq i \leq m(S)} t\left(P_{S, i}\right) \leq c_{4} \Delta(S)$.

5. Auxiliary lemmas. The proof of the following lemma is easy, and hence we shall omit it.

Lemma 1. For every polynomial $P \in \mathbb{C}\left[X_{1}, \ldots, X_{t}\right]$ and for any two points $\underline{z}=\left(z_{1}, \ldots, z_{t}\right)$ and $\underline{z}^{\prime}=\left(z_{1}^{\prime}, \ldots, z_{t}^{\prime}\right)$ of $\mathbb{C}^{t}$ satisfying $\max _{1 \leq i \leq t}\left|z_{i}-z_{i}^{\prime}\right| \leq$ $\varepsilon<1$, we have

$$
\left|P(\underline{z})-P\left(\underline{z}^{\prime}\right)\right| \leq \varepsilon \exp \left(c^{\prime} t(P)\right),
$$

where $c^{\prime}>1$ depends only on $\underline{z}$ and $t$.

Lemma 2. For all $\underline{h} \in \mathbb{Z}^{m}(S)$, there exist a finite set $\mathcal{B}_{\underline{h}}$ and a family $\left(u_{i}^{\beta}\right)_{\beta \in \mathcal{B}_{\underline{h}}, 0 \leq i \leq N}$ of polynomials in $q+1$ variables with integral coefficients in $K$ such that

(i) $t\left(u_{i}^{\beta}\right) \leq c_{5} S^{2}$,

(ii) for every $\underline{\tilde{\theta}} \in B^{q}(\underline{\theta}, \exp (-c \varrho(S)))$, there exists $\beta \in \mathcal{B}_{\underline{h}}$ such that $\left(u_{0}^{\beta}(\underline{\tilde{\theta}}), \ldots, u_{N}^{\beta}(\underline{\widetilde{\theta}})\right)$ is a system of projective coordinates of $\chi_{2} \circ$ $\exp _{G_{2}}\left(p_{2}(\underline{h} \cdot \underline{\widetilde{y}})\right)$.

Proof. For the polynomials $\left(U_{i}^{\beta}\right)_{\beta \in \mathcal{B}_{h}, 0 \leq i \leq N}$ in [1, lemme 2.2], put

$$
\begin{aligned}
& u_{i}^{\beta}(\underline{Y})= \\
& \quad U_{i}^{\beta}\left(Q(\underline{Y}), D_{1,1}(\underline{Y}), \ldots, D_{N, 1}(\underline{Y}), \ldots, Q(\underline{Y}), D_{1, m-\ell}(\underline{Y}), \ldots, D_{N, m-\ell}(\underline{Y})\right) .
\end{aligned}
$$


Lemma 3 ([19, Proposition 1.2.3]). Suppose that $T_{G}(\mathbb{C})$ is identified with $\mathbb{C}^{d}=\left\{\left(z_{1}, \ldots, z_{d}\right) ; z_{i} \in \mathbb{C}\right\}$. Let $\Theta_{0}, \ldots, \Theta_{N}$ be as in $\S 2$. Then if $\Theta_{j} \neq 0$ $(0 \leq j \leq N)$, there exist polynomials $Q_{i s}(1 \leq i \leq d, 0 \leq s \leq N, s \neq j)$, depending on $j$, with coefficients in $K$ such that

$$
\frac{\partial}{\partial z_{i}}\left(\frac{\Theta_{s}}{\Theta_{j}}\right)=Q_{i s}\left(\frac{\Theta_{0}}{\Theta_{j}}, \ldots, \frac{\Theta_{s-1}}{\Theta_{j}}, \frac{\Theta_{s+1}}{\Theta_{j}}, \ldots, \frac{\Theta_{N}}{\Theta_{j}}\right) .
$$

6. Proof of Proposition B. In the preceding notation, recall $\underline{a}_{1}=$ $\left(a_{11}, \ldots, a_{d 1}\right), \ldots, \underline{a}_{n}=\left(a_{1 n}, \ldots, a_{d n}\right)$. Now we define $n$ differential operators $D_{\underline{a}_{1}}, \ldots, D_{\underline{a}_{n}}$ by

$$
D_{\underline{a}_{1}}=\sum_{i=1}^{d} a_{i 1} \frac{\partial}{\partial z_{i}}, \ldots, D_{\underline{a}_{n}}=\sum_{i=1}^{d} a_{i n} \frac{\partial}{\partial z_{i}} .
$$

For $S \geq S_{1}$, we consider a finite set $\mathcal{B}_{\underline{h}}$ and a family $\left(u_{i}^{\beta}\right)\left(\beta \in \mathcal{B}_{\underline{h}}, 0 \leq i \leq N\right)$ of polynomials given in Lemma 2 . Then the proof depends on the quantity $\max _{\beta \in \mathcal{B}_{\underline{h}}, 0 \leq i \leq N}\left|u_{i}^{\beta}(\underline{\theta})\right|$.

CASE 1: There exists $\underline{h} \in \mathbb{Z}^{m}(S)$ such that

$$
\max _{\beta \in \mathcal{B}_{\underline{h}}, 0 \leq i \leq N}\left|u_{i}^{\beta}(\underline{\theta})\right| \leq \exp \left(-\frac{c}{5} \frac{\varrho(S)}{D_{2}(S)-1}\right) .
$$

Since the proof of this case is as in Ably [1], we shall omit it.

CASE 2: For all $\underline{h} \in \mathbb{Z}^{m}(S)$,

$$
\max _{\beta \in \mathcal{B}_{\underline{h}}, 0 \leq i \leq N}\left|u_{i}^{\beta}(\underline{\theta})\right|>\exp \left(-\frac{c}{5} \frac{\varrho(S)}{D_{2}(S)-1}\right) .
$$

In a similar fashion to that of Ably [1], we divide the argument into several steps. In the first step, we shall construct an auxiliary function with many zeros, by Siegel's lemma and the estimation of rank in $[14$, lemme 6.7]. In the second step, we use the idea due to G. Diaz [7] to construct an ideal $\mathcal{I}_{S}$ which takes "small values" at $\underline{\theta}$ by the extrapolation formula. In the third step, we appeal to P. Philippon's zero estimate [13] on algebraic groups to show that the variety of zeros of $\mathcal{I}_{S}$ is locally empty.

STEP 1: Construction of an auxiliary function. For $S \geq S_{1}$, we put

$$
M(S)=\left[\frac{1}{c^{d+1}} S^{(\kappa-1) \mu^{\sharp} / m}\right], \quad T(S)=\left[S^{\mu^{\sharp}}(\log S)^{a-1}\right] .
$$

Note that $M(S) \geq S_{0}$, since $S \geq S_{1}$, and that $M(S)<S$, because $(\kappa-1) \mu^{\sharp}$ $\leq m$ and $c$ is sufficiently large. For simplicity, we write $D_{0}, D_{1}, D_{2}, A, B$, $M, \ldots$ instead of $D_{0}(S), D_{1}(S), D_{2}(S), A(S), B(S), M(S), \ldots$ 
Let $E$ be a set of monic monomials in $\underline{Z}$ with degree $D_{2}$, linearly independent over $K(\underline{\theta})$ modulo the homogeneous ideal $I$ of $K(\underline{\theta})[\underline{Z}]$ of polynomials which vanish on $G_{2}$; since the $K(\underline{\theta})$-vector space of elements of $K(\underline{\theta})[\underline{Z}] / I$ of degree $D_{2}-1$ is of dimension $\geq c_{6} D_{2}^{d_{2}}$, we can take $E$ such that card $E \geq c_{6} D_{2}^{d_{2}}$.

We consider the following polynomial:

$$
P(X, \underline{Y}, \underline{Z})=\sum_{\alpha \leq D_{0}-1} \sum_{|\underline{\beta}| \leq D_{1}-1} \sum_{\underline{Z} \underline{\lambda} \in E} P_{\alpha \underline{\beta} \underline{\lambda}}(\underline{\theta}) X^{\alpha} Y_{1}^{\beta_{1}} \cdots Y_{d_{1}}^{\beta_{d_{1}}} Z_{0}^{\lambda_{0}} \cdots Z_{N}^{\lambda_{N}},
$$

where $\underline{\beta}=\left(\beta_{1}, \ldots, \beta_{d_{1}}\right), \underline{\lambda}=\left(\lambda_{0}, \ldots, \lambda_{N}\right),|\underline{\beta}|=\beta_{1}+\cdots+\beta_{d_{1}}, \underline{Z} \underline{\underline{\lambda}}=$ $Z_{0}^{\lambda_{0}} \cdots \bar{Z}_{N}^{\lambda_{N}}$, and $P_{\alpha \underline{\beta} \underline{\lambda}} \in \mathbb{Z}\left[X_{1}, \ldots, X_{q}\right]$ and $P_{\alpha \underline{\beta} \underline{\lambda}}(\underline{\theta})=P_{\alpha \underline{\beta} \underline{\lambda}}\left(\theta_{1}, \ldots, \theta_{q}\right)$.

We put $\underline{z}=\left(z_{0}, z_{1}, \ldots, z_{d_{1}}, z_{d_{1}+1}, \ldots, z_{d_{1}+d_{2}}\right)$ and $\underline{z}^{\prime}=\left(z_{d_{1}+1}, \ldots, z_{d_{1}+d_{2}}\right)$. Now we define a function $\Psi: \mathbb{C}^{d} \rightarrow \mathbb{C}^{2+d_{1}+N}$ by

$$
\Psi(\underline{z})=\left(z_{0}, e^{z_{1}}, \ldots, e^{z_{d_{1}}}, \Theta_{0}\left(\underline{z}^{\prime}\right), \ldots, \Theta_{N}\left(\underline{z}^{\prime}\right)\right) .
$$

We put

$$
\begin{aligned}
& F(\underline{z}):=P(\Psi(\underline{z}))=\sum_{\substack{\alpha \leq D_{0}-1 \\
|\underline{\beta}| \leq D_{1}-1 \\
\underline{Z^{\lambda}} \in E}} P_{\alpha \underline{\beta} \underline{\lambda}}(\underline{\theta}) z_{0}^{\alpha} \exp \left(\beta_{1} z_{1}+\cdots+\beta_{d_{1}} z_{d_{1}}\right) \\
& \times \Theta_{0}\left(\underline{z}^{\prime}\right)^{\lambda_{0}} \cdots \Theta_{N}\left(\underline{z}^{\prime}\right)^{\lambda_{N}} .
\end{aligned}
$$

By Philippon [11], there exist polynomials $A_{0}, \ldots, A_{N}$ bihomogeneous in $\left(X_{0}, \ldots, X_{N} ; X_{0}^{\prime}, \ldots, X_{N}^{\prime}\right)$ such that

$$
\Theta_{0}\left(\underline{z}^{\prime}+\underline{u}^{\prime}\right)=A_{0}\left(\Psi^{\prime}\left(\underline{z}^{\prime}\right) ; \Psi^{\prime}\left(\underline{u}^{\prime}\right)\right), \ldots, \Theta_{N}\left(\underline{z}^{\prime}+\underline{u}^{\prime}\right)=A_{N}\left(\Psi^{\prime}\left(\underline{z}^{\prime}\right) ; \Psi^{\prime}\left(\underline{u}^{\prime}\right)\right)
$$

for $\underline{z}^{\prime}$ and $\underline{u}^{\prime}$ in $\mathbb{C}^{d_{2}}$, where $\Psi^{\prime}\left(\underline{z}^{\prime}\right)=\left(\Theta_{0}\left(\underline{z}^{\prime}\right), \ldots, \Theta_{N}\left(\underline{z}^{\prime}\right)\right)$. Hence we have

$$
\begin{aligned}
F(\underline{z}+\underline{u})=\sum_{\substack{\alpha \leq D_{0}-1 \\
\mid \underline{\beta} \leq \leq D_{1}-1 \\
\underline{Z^{\lambda} \in E}}} P_{\alpha \underline{\beta} \underline{\lambda}}(\underline{\theta})\left(z_{0}+u_{0}\right)^{\alpha} \exp \left(\beta_{1}\left(z_{1}+u_{1}\right)+\cdots+\beta_{d_{1}}\left(z_{d_{1}}+u_{d_{1}}\right)\right) \\
\times A_{0}\left(\Psi^{\prime}\left(\underline{z}^{\prime}\right), \Psi^{\prime}\left(\underline{u}^{\prime}\right)\right)^{\lambda_{0}} \cdots A_{N}\left(\Psi^{\prime}\left(\underline{z}^{\prime}\right), \Psi^{\prime}\left(\underline{u}^{\prime}\right)\right)^{\lambda_{N}}
\end{aligned}
$$

for $\underline{z}=\left(z_{0}, \ldots, z_{d_{1}+d_{2}}\right)$ and $\underline{u}=\left(u_{0}, \ldots, u_{d_{1}+d_{2}}\right)$. Then for $\underline{h} \in \mathbb{N}^{m}(S)$, we obtain

$$
F(\underline{z}+\operatorname{Lie} \varphi(\underline{h} \cdot \underline{y}))=\sum_{\substack{\alpha \leq D_{0}-1 \\|\underline{\beta}| \leq D_{1}-1 \\ \underline{Z}^{\lambda} \in E}} P_{\alpha \underline{\beta} \underline{\lambda}}(\underline{\theta}) R_{\alpha \underline{\beta} \underline{\lambda}}(\Psi(\underline{z}) ; \Psi(\operatorname{Lie} \varphi(\underline{h} \cdot \underline{y}))),
$$

where $R_{\alpha \underline{\beta} \underline{\lambda}}\left(X, \underline{Y}, \underline{Z} ; X^{\prime}, \underline{Y}^{\prime}, \underline{Z}^{\prime}\right)$ is a polynomial in $\left(X, \underline{Y}, \underline{Z} ; X^{\prime}, \underline{Y}^{\prime}, \underline{Z}^{\prime}\right)$ with $\operatorname{deg}_{X, X^{\prime}}, R_{\alpha \underline{\beta} \underline{\lambda}} \leq \alpha \leq D_{0}-1, \operatorname{deg}_{\underline{Y}, \underline{Y}^{\prime}} R_{\alpha \underline{\beta} \underline{\lambda}} \leq|\underline{\beta}| \leq D_{1}-1$, and $\operatorname{deg}_{\underline{Z}, \underline{Z}^{\prime}} R_{\alpha, \underline{\beta} \underline{\lambda}}$ $\leq c_{7}\left(\lambda_{0}+\cdots+\lambda_{n}\right) \leq c_{7} D_{2}$. Note that

$$
D_{\underline{a}_{1}}^{t_{1}} \cdots D_{\underline{a}_{n}}^{t_{n}} F(\underline{z}+\underline{u})_{\underline{z}=0}=D_{\underline{a}_{1}}^{t_{1}} \cdots D_{\underline{a}_{n}}^{t_{n}} F(\underline{u})
$$


for $\left(t_{1}, \ldots, t_{n}\right) \in \mathbb{N}^{n}$ and $\underline{u} \in \mathbb{C}^{d}$. For each $\underline{h} \in \mathbb{N}^{m}(S)$ and $\Theta_{j_{0}}\left(\underline{z}^{\prime}\right) \neq 0$, we see from Lemma 3 that

$$
\begin{aligned}
D_{\underline{a}_{1}}^{t_{1}} \cdots D_{\underline{a}_{n}}^{t_{n}}\left(\frac{F(\underline{z}+\operatorname{Lie} \varphi(\underline{h} \cdot \underline{y}))}{\Theta_{j_{0}}\left(\underline{z}^{\prime}\right)^{c_{8} D_{2}}}\right)_{\underline{z}=0}=\sum_{\substack{\alpha \leq D_{0}-1 \\
|\underline{\mid \beta}| \leq D_{1}-1}} P_{\alpha \underline{\beta} \underline{\lambda}}(\underline{\theta}) \\
\quad \times U_{\alpha \underline{\underline{\beta}} \underline{\lambda}}\left(\underline{h} \cdot \underline{y}, \underline{\underline{y}}, \underline{a}_{1}, \ldots, \underline{a}_{n}, e^{x_{1}(\underline{h} \cdot \underline{y})}, \ldots, e^{x_{d_{1}}(\underline{h} \cdot \underline{y})}, \Theta_{0}(\operatorname{Lie} \psi(\underline{h} \cdot \underline{y})), \ldots\right. \\
\left.\ldots, \Theta_{N}(\operatorname{Lie} \psi(\underline{h} \cdot \underline{y}))\right),
\end{aligned}
$$

where $U_{\alpha \underline{\beta} \underline{\lambda}}$ is a polynomial with algebraic coefficients in variables $\left(X, V_{01}, \ldots\right.$ $\left.\ldots, V_{d_{1}+d_{2}, 1}, \ldots, V_{0 n}, \ldots, V_{d_{1}+d_{2}, n}, \underline{Y}, \underline{Z}\right)$ with $\operatorname{deg}_{X} U_{\alpha \underline{\beta} \underline{\lambda}} \leq \alpha \leq D_{0}-1$, $\operatorname{deg}_{V} U_{\alpha \underline{\beta} \underline{\lambda}} \leq|\underline{t}| \leq T, \operatorname{deg}_{\underline{Y}} U_{\alpha \underline{\beta} \underline{\lambda}} \leq\left(h_{1}+\cdots+h_{m}\right) \cdot \max \beta_{i} \leq m D_{1} S$, $\operatorname{deg}_{\underline{Z}} U_{\alpha \underline{\beta} \underline{\underline{\lambda}}} \leq c_{9} D_{2}$, and $t\left(U_{\alpha \underline{\beta} \underline{\underline{\lambda}}}\right) \leq c_{10} T \log \left(D_{0}+D_{1}+D_{2}+T\right)$.

For each $\underline{h} \in \mathbb{N}^{m}(S)$, we choose $j_{\underline{h}}, 0 \leq j_{\underline{h}} \leq N, \beta_{\underline{h}} \in \mathcal{B}_{\underline{h}}$ such that

$$
\max _{\beta \in \mathcal{B}_{\underline{h}}, 0 \leq i \leq N}\left|u_{i}^{\beta}(\underline{\theta})\right|=\left|u_{j_{\underline{h}}}^{\beta_{\underline{h}}}(\underline{\theta})\right| .
$$

Here $\left(u_{0}^{\beta_{\underline{h}}}(\underline{\theta}), \ldots, u_{N}^{\beta_{h}}(\underline{\theta})\right)$ are the projective coordinates of $\chi_{2} \circ \psi(\underline{h} \cdot \underline{y})$. Then we have

$$
\begin{aligned}
& Q(\underline{\theta})^{T+D_{0}+m D_{1} S}\left(\frac{u_{\underline{\underline{h}}}^{\beta_{\underline{h}}}(\underline{\theta})}{\Theta_{j_{\underline{h}}}(\operatorname{Lie} \psi(\underline{h} \cdot \underline{y}))}\right)^{c_{8} D_{2}} \\
& \times D_{\underline{a}_{1}}^{t_{1}} \cdots D_{\underline{a}_{n}}^{t_{n}}\left(\frac{F(\underline{z}+\operatorname{Lie} \varphi(\underline{h} \cdot \underline{y}))}{\Theta_{j_{0}}\left(\underline{z}^{\prime}\right)^{c_{8} D_{2}}}\right)_{\underline{z}=0}=\sum_{\substack{\alpha \leq D_{0}-1 \\
|\underline{\beta}| \leq D_{1}-1 \\
\underline{Z}^{\lambda} \in E}} P_{\alpha \underline{\beta} \underline{\lambda}}(\underline{\theta}) H_{\alpha \underline{\beta} \underline{\lambda} \underline{\underline{h}} \underline{\underline{h}}}(\underline{\theta}),
\end{aligned}
$$

where $H_{\alpha \underline{\beta} \underline{\underline{\lambda}} \underline{\underline{t}} \underline{t}}\left(X_{1}, \ldots, X_{q+1}\right)$ is a polynomial with integer coefficients in $K$.

Now we shall require that $D_{0} \sim T$. For this we shall choose a constant $a$ (cf. $§ 2$ ) as follows:

$$
(a-1) n+d_{0}=a d,
$$

and hence $a=-\left(n-d_{0}\right) /(d-n)<0$. Then we have

$$
\begin{aligned}
t\left(H_{\alpha \underline{\beta} \underline{\lambda} \underline{h} \underline{t}}\right) & \leq c_{11}\left(D_{0} \log S+\left(T \wedge D_{0}\right) \log D_{0}+T \log D_{1}+D_{1} S+D_{2} S^{2}\right) \\
& \leq c_{12} \Delta(S),
\end{aligned}
$$

where $T \wedge D_{0}$ means $\min \left\{T, D_{0}\right\}$. We put

$$
\begin{aligned}
& \underline{X}=\left(X_{1}, \ldots, X_{q+1}\right), \quad P_{\alpha \underline{\beta} \underline{\lambda}}(\underline{X})=P_{\alpha \underline{\beta} \underline{\lambda}}\left(X_{1}, \ldots, X_{q}\right), \\
& H_{\underline{h} \underline{t}}(\underline{X})=\sum_{\alpha \leq D_{0}-1} \sum_{|\underline{\beta}| \leq D_{1}-1} \sum_{\underline{Z} \underline{\lambda} \in E} P_{\alpha \underline{\beta} \underline{\lambda}}(\underline{X}) H_{\alpha \underline{\beta} \underline{\lambda} \underline{h} \underline{t}}(\underline{X}), \\
& n_{i}=\max \left\{\operatorname{deg}_{X_{i}} H_{\alpha \underline{\beta} \underline{\lambda} \underline{h} \underline{t}} ; \alpha \leq D_{0}-1,|\underline{\beta}| \leq D_{1}-1\right. \text {, } \\
& \left.\underline{Z}^{\underline{\lambda}} \in E, \underline{h} \in \mathbb{N}^{m}(M),|\underline{t}|<T\right\}+1 \quad(1 \leq i \leq q) .
\end{aligned}
$$


The purpose of this first step is to find polynomials $P_{\alpha \underline{\beta} \underline{\lambda}}$ not all 0 with rational integral coefficients with $\operatorname{deg}_{X_{i}} P_{\alpha \underline{\beta} \underline{\lambda}} \leq n_{i}(1 \leq \bar{i} \leq q)$ such that $H_{\underline{h} \underline{t}}(\underline{X})=0$ for all $\underline{h} \in \mathbb{N}^{m}(M)$ and for all $\underline{t} \in \mathbb{N}^{n},|\underline{t}|<T$. Now we consider the system

$$
\left\{H_{\underline{h} \underline{\underline{t}}}(\underline{X})=0 ; \underline{h} \in \mathbb{N}^{m}(M), \underline{t} \in \mathbb{N}^{n},|\underline{t}|<T\right\}
$$

with unknowns the coefficients of the polynomials $P_{\alpha \underline{\beta}} \underline{\lambda}\left(\alpha \leq D_{0}-1,|\underline{\beta}| \leq\right.$ $\left.D_{1}-1, \underline{Z}^{\underline{\lambda}} \in E\right)$ and with coefficients the coefficients of the polynomials $H_{\alpha \underline{\beta} \underline{\lambda} \underline{h} \underline{t} j}\left(\alpha \leq D_{0}-1,|\underline{\beta}| \leq D_{1}-1, \underline{Z} \underline{\lambda} \in E, \underline{h} \in \mathbb{N}^{m}(M),|\underline{t}|<T\right.$, $\left.0 \leq j \leq \delta^{\prime}-1\right)$. Then we shall show that $\left(\mathcal{S}_{1}\right)$ has a nontrivial solution. The system of linear equations

$$
\left\{D_{\underline{a}_{1}}^{t_{1}} \cdots D_{\underline{a}_{n}}^{t_{n}} F(\operatorname{Lie} \varphi(\underline{h} \cdot \underline{y}))=0 ; \underline{h} \in \mathbb{N}^{m}(M), \underline{t} \in \mathbb{N}^{n},|\underline{t}|<T\right\}
$$

with unknowns $x_{\tau}$ with $\left\{x_{\tau} ; \tau\right\}=\left\{P_{\alpha \underline{\beta} \underline{\lambda}}(\underline{\theta}) ; \alpha \leq D_{0}-1,|\underline{\beta}| \leq D_{1}-1, \underline{Z} \underline{\lambda} \in E\right\}$ is of rank at most $8^{\operatorname{dim} G^{\prime}} T^{\nu} \operatorname{card}\left(\left(\Gamma(M)+G^{\prime}\right) / G^{\prime}\right) H\left(G^{\prime} ; D_{0}, D_{1}, D_{2}\right)$ for every connected algebraic subgroup $G^{\prime} \subsetneq G$ (cf. [14, lemma 6.7]). By (4), $\left(\mathcal{S}_{2}\right)$ is equivalent to the system of linear equations

$$
\left\{H_{\underline{h} \underline{t}}(\underline{\theta})=0 ; \underline{h} \in \mathbb{N}^{m}(M), \underline{t} \in \mathbb{N}^{n},|\underline{t}|<T\right\}
$$

with unknowns $x_{\tau}$.

On the other hand, we have

$$
H_{\underline{h} \underline{t}}(\underline{\theta})=0 \Leftrightarrow \sum_{\substack{\alpha \leq D_{0}-1 \\|\underline{\beta}| \leq D_{1}-1 \\ \underline{Z} \underline{\lambda}^{\lambda} \in E}} P_{\alpha \underline{\beta} \underline{\lambda}}(\underline{\theta}) H_{\alpha \underline{\beta} \underline{\lambda} \underline{h} \underline{t} j}(\theta)=0\left(\forall j, 0 \leq j \leq \delta^{\prime}-1\right) .
$$

Since $\theta_{1}, \ldots, \theta_{q}$ are algebraic independent over $K$, we obtain a linear system such that the unknowns are the coefficients of $P_{\alpha \underline{\beta} \underline{\underline{\lambda}}}$ and the coefficients are the coefficients of $H_{\alpha \underline{\beta}} \underline{\underline{\lambda}} \underline{\underline{t}} \underline{j} j$, which is exactly the system $\left(\mathcal{S}_{1}\right)$.

From $\operatorname{deg}_{X_{i}} P_{\alpha \underline{\beta} \underline{\lambda}} \leq n_{i}$ and $\operatorname{deg}_{X_{i}} H_{\alpha \underline{\beta} \underline{\lambda} \underline{\underline{h}} \underline{\underline{t}} \leq} \leq n_{i}(1 \leq i \leq q)$, we deduce that the rank $L^{\prime}$ of $\left(\mathcal{S}_{1}\right)$ satisfies $L^{\prime} \leq 2^{q}\left(\prod_{i=1}^{q} n_{i}\right) \delta^{\prime} \cdot \operatorname{rank}\left(\mathcal{S}_{2}\right)$. From the same arguments as in Ably [1], we deduce

$$
\begin{aligned}
L^{\prime} \leq 2^{q}\left(\prod_{i=1}^{q} n_{i}\right) \operatorname{deg}_{X_{q+1}} R \\
\quad \times 8^{\operatorname{dim} G^{\prime}} T^{\nu} \operatorname{card}\left(\left(\Gamma(M)+G^{\prime}\right) / G^{\prime}\right) H\left(G^{\prime} ; D_{0}, D_{1}, D_{2}\right) .
\end{aligned}
$$

The number $N$ of the unknowns of $\left(\mathcal{S}_{1}\right)$ is at least $c_{13}\left(\prod_{i=1}^{q} n_{i}\right) D_{0}^{d_{0}} D_{1}^{d_{1}} D_{2}^{d_{2}}$. We shall show that $L^{\prime}<N$. Recall the definition of $A$ and $B$ in $\S 2$. tain

CAse (i): $B=c^{-1}(\log S)^{\left((a-1) n+d_{0}\right) / d}$. Taking $G^{\prime}=\{0\}$ in (5), we ob-

$$
L^{\prime} \leq 2^{q}\left(\prod_{i=1}^{q} n_{i}\right) \operatorname{deg}_{X_{q+1}} R \cdot T^{n} M^{m-\ell},
$$


since $\nu=n$. On the other hand, it follows from our choices of parameters that

$$
D_{0}^{d_{0}} D_{1}^{d_{1}} D_{2}^{d_{2}} \geq \frac{1}{2 c^{d}} S^{\mu^{\sharp} d-d_{1}-2 d_{2}}(\log S)^{(a-1) n} .
$$

Recalling the definition of $T$ and $M$ and combining these results, we have $N \geq 2[K: \mathbb{Q}] L^{\prime}$, since $c$ is sufficiently large.

CASE (ii): $B=A$. Let $G_{0}^{\prime}$ be the connected algebraic subgroup of $G$ such that $A=A\left(S, G_{0}^{\prime}\right)$. From the definitions of $A\left(S, G_{0}^{\prime}\right)$ and the parameters $\bar{D}_{0}, \bar{D}_{1}, \bar{D}_{2}$, we have

$$
A^{\operatorname{dim} G / G_{0}^{\prime}} \geq \frac{1}{c} T^{\nu} \operatorname{card}\left(\left(\Gamma(S)+G_{0}^{\prime}\right) / G_{0}^{\prime}\right) \frac{H\left(G_{0}^{\prime} ; \bar{D}_{0} / B, \bar{D}_{1} / B, \bar{D}_{2} / B\right)}{H\left(G ; \bar{D}_{0} / B, \bar{D}_{1} / B, \bar{D}_{2} / B\right)} .
$$

Taking account of the homogeneity of $H$ and noting $A=B$, we have

$$
H\left(G ; \bar{D}_{0}, \bar{D}_{1}, \bar{D}_{2}\right) \geq \frac{1}{c} T^{\nu} \operatorname{card}\left(\left(\Gamma(S)+G_{0}^{\prime}\right) / G_{0}^{\prime}\right) H\left(G_{0}^{\prime} ; \bar{D}_{0}, \bar{D}_{1}, \bar{D}_{2}\right) .
$$

Furthermore, using (1) and recalling the choice of $M$ we have

$$
\begin{aligned}
D_{0}^{d_{0}} D_{1}^{d_{1}} D_{2}^{d_{2}}>\frac{1}{c_{14}} 2^{q+1} & \left.\operatorname{deg}_{X_{q+1}} R\right) 8^{\operatorname{dim} G_{0}^{\prime}}[K: \mathbb{Q}] T^{\nu} \\
& \times \operatorname{card}\left(\left(\Gamma(M)+G_{0}^{\prime}\right) / G_{0}^{\prime}\right) H\left(G_{0}^{\prime} ; D_{0}, D_{1}, D_{2}\right),
\end{aligned}
$$

where $c_{14}=c_{13}\left(\prod n_{i}\right)$, since $c$ is sufficiently large. Finally, taking $G^{\prime}=G_{0}^{\prime}$ in (5), we obtain $N \geq 2[K: \mathbb{Q}] L^{\prime}$.

Therefore in both cases, we have the same upper bound, and hence we can apply Siegel's lemma to find a nontrivial solution of the system $\left(\mathcal{S}_{1}\right)$ such that $\max \left(t\left(P_{\alpha \underline{\beta} \underline{\lambda}}\right)\right) \leq c_{15} \Delta(S)$.

SteP 2: Derivation of coefficients and extrapolation. The polynomials $H_{\underline{h} \underline{\underline{t}}}$ constructed in the first step may vanish in a neighborhood of $\underline{\theta}$, and hence we need to modify them to get polynomials that satisfy the conditions of the proposition. For this we shall make use of the idea of Chudnovsky, developed by Diaz [7]. For $\underline{i}=\left(i_{1}, \ldots, i_{q}\right) \in \mathbb{N}^{q}$ we define the differential operator and the length of $\underline{i}$ by

$$
D^{\underline{i}}=\frac{1}{i_{1} ! \cdots i_{q} !}\left(\frac{\partial}{\partial X_{1}}\right)^{i_{1}} \cdots\left(\frac{\partial}{\partial X_{q}}\right)^{i_{q}}, \quad|\underline{i}|=i_{1}+\cdots+i_{q},
$$

respectively. Let $\underline{\tilde{\theta}} \in B^{q}(\underline{\theta}, \exp (-c \varrho(S)))$, and let $\left(\widetilde{\theta}_{1}, \ldots, \widetilde{\theta}_{q+1}\right)$ be the element of $\mathbb{C}^{q+1}$ associated to $\underline{\tilde{\theta}}$ as in $\S 2$; we denote it by the same notation again. The set

$I(\underline{\widetilde{\theta}}):=\left\{\underline{i} \in \mathbb{N}^{q} ; \exists(\alpha, \underline{\beta}, \underline{\lambda}), \alpha \leq D_{0}-1,|\underline{\beta}| \leq D_{1}-1, \underline{Z}^{\underline{\lambda}} \in E, D^{\underline{i}} P_{\alpha \underline{\beta}} \underline{\lambda}(\underline{\widetilde{\theta}}) \neq 0\right\}$ is nonempty and finite. Put

$$
\underline{i}(\underline{\widetilde{\theta}}):=\min _{\underline{i} \in I(\underline{\widetilde{\theta}})}|\underline{i}|, \quad I:=\left\{\underline{i}(\underline{\widetilde{\theta}}) ; \underline{\tilde{\theta}} \in B^{q}(\underline{\theta}, \exp (-c \varrho(S)))\right\} .
$$


For $\underline{i} \in I$, we put

$$
H_{\underline{h} \underline{t} \underline{i}}(\underline{X})=\sum_{\alpha \leq D_{0}-1} \sum_{|\underline{\beta}| \leq D_{1}-1} \sum_{\underline{Z} \underline{\lambda} \in E} D^{\underline{i}} P_{\alpha \underline{\beta}} \underline{\lambda}(\underline{X}) H_{\alpha \underline{\beta} \underline{\lambda} \underline{h} \underline{\underline{t}}}(\underline{X}) .
$$

Lemma 4. For all $\underline{h} \in \mathbb{N}^{m}(S)$, all $\underline{t} \in \mathbb{N}^{n}$ with $|\underline{t}|<T / 2$, and all $\underline{i} \in I$, we have

$$
\left|H_{\underline{h} \underline{\underline{t}} \underline{i}}(\underline{\theta})\right| \leq \exp \left(-c_{16} \varrho(S)\right) .
$$

Proof. Following Ably [1], we first prove this for $\underline{h} \in \mathbb{N}^{m}(M)$, and then for $\underline{h} \in \mathbb{N}^{m}(S)$. We fix $\underline{i} \in I$ and $\underline{\tilde{\theta}}$ such that $\underline{i}(\underline{\widetilde{\theta}})=\underline{i}$. We have

$$
\begin{aligned}
& H_{\underline{h} \underline{t} \underline{i}}(\underline{\theta})=\sum_{\substack{\alpha \leq D_{0}-1 \\
|\underline{\beta}| \leq D_{1}-1 \\
\underline{Z} \underline{\lambda}^{\underline{\lambda}} \in E}}\left(D^{\underline{i}} P_{\alpha \underline{\beta} \underline{\lambda}}(\underline{\theta})-D^{\underline{i}} P_{\alpha \underline{\beta}} \underline{\lambda}(\underline{\theta})\right) H_{\alpha \underline{\beta}} \underline{\lambda} \underline{\underline{h}} \underline{t}(\underline{\theta})
\end{aligned}
$$

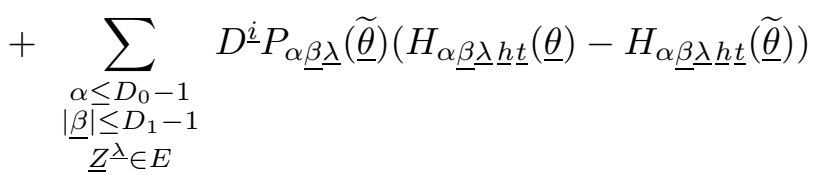

$$
\begin{aligned}
& +\sum_{\substack{\alpha \leq D_{0}-1 \\
|\underline{\beta}| \leq D_{1}-1 \\
\underline{Z} \underline{\lambda} \in E}} D^{\underline{i}} P_{\alpha \underline{\beta} \underline{\lambda}}(\underline{\widetilde{\theta}}) H_{\alpha \underline{\beta} \underline{\lambda} \underline{\lambda} \underline{t} \underline{t}(\underline{\widetilde{\theta}}) .}
\end{aligned}
$$

By the definition of $\underline{i}=\underline{i}(\underline{\widetilde{\theta}})$ and the construction of $H_{\underline{h} \underline{t}}$, we have

$$
D^{\underline{i}} H_{\underline{h} \underline{t}}(\underline{\widetilde{\theta}})=\sum_{\alpha \leq D_{0}-1} \sum_{|\underline{\beta}| \leq D_{1}-1} \sum_{\underline{Z} \underline{\lambda} \in E} D^{\underline{i}} P_{\alpha \underline{\beta} \underline{\lambda}}(\underline{\widetilde{\theta}}) H_{\alpha \underline{\beta} \underline{\lambda} \underline{h} \underline{\underline{t}}}(\underline{\widetilde{\theta}})=0 .
$$

Using this and Lemma 1 in (6), we have

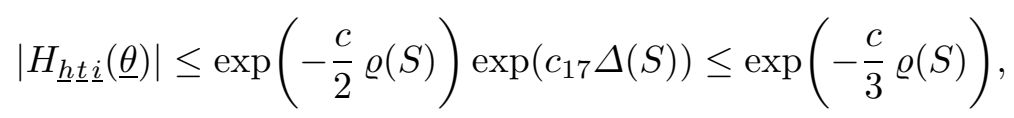

because $t\left(H_{\alpha \underline{\beta} \underline{\lambda} \underline{h} \underline{\underline{t}}}\right) \leq c_{12} \Delta(S)$ and $t\left(D^{\underline{i}} P_{\alpha \underline{\beta} \underline{\lambda}}\right) \leq c_{18} \Delta(S)$.

Extrapolation. We shall extend this upper bound to the pair $(\underline{h}, \underline{t})$ with $\underline{h} \in \mathbb{N}^{m}(S), \underline{t} \in \mathbb{N}^{n},|\underline{t}|<T / 2$. For this we consider the following polynomial and an analytic function:

$$
\begin{aligned}
\widetilde{P}(X, \underline{Y}, \underline{Z}) & =\sum_{\alpha \leq D_{0}-1} \sum_{|\underline{\beta}| \leq D_{1}-1} \sum_{\underline{Z} \underline{\underline{\lambda}} \in E} D^{\underline{\underline{i}}} P_{\alpha \underline{\beta} \underline{\lambda}}(\underline{\widetilde{\theta}}) X^{\alpha} \underline{Y}^{\underline{\beta}} \underline{Z}^{\underline{\lambda}}, \\
\widetilde{F}(\underline{z}) & =\widetilde{P}(\Psi(\underline{z})),
\end{aligned}
$$

where $\underline{z}=\left(z_{0}, z_{1}, \ldots, z_{d_{1}}, z_{d_{1}+1}, \ldots, z_{d_{1}+d_{2}}\right)=\left(z_{0}, z_{1}, \ldots, z_{d_{1}}, \underline{z}^{\prime}\right)$. We put $R_{1}(S)=c_{19} S$ with $c_{19}=\max _{j}\left\{2, m\left|y_{j}\right|\right\}$, and $R_{2}(S)=S^{1+(\kappa-1) \mu^{\sharp} / 3}$. For 
simplicity, we write $M, R_{1}$ and $R_{2}$ in place of $M(S), R_{1}(S)$ and $R_{2}(S)$. We define a one-variable function $\widetilde{g}_{\underline{t}}(z)$ by

$$
\widetilde{g}_{\underline{t}}(z)=D_{\underline{a}_{1}}^{t_{1}} \cdots D_{\underline{a}_{n}}^{t_{n}} \widetilde{F}(\operatorname{Lie} \varphi(z))
$$

for $\underline{t}=\left(t_{1}, \ldots, t_{n}\right) \in \mathbb{N}^{n}$; this is clearly an entire function. We define

$$
\delta(M)=\min _{\substack{y^{\prime}, y^{\prime \prime} \in Y(M) \\ y^{\prime} \neq y^{\prime \prime}}}\left\{1,\left|y^{\prime}-y^{\prime \prime}\right|\right\} .
$$

By applying an extrapolation formula [15, lemme 4.5] to the function $\widetilde{g}_{\underline{t}}(z)$, we obtain

(9) $\left|\widetilde{g}_{\underline{t}}\right|_{R_{1}} \leq 2\left|\widetilde{g}_{\underline{t}}\right|_{R_{2}}\left(\frac{4 R_{1}}{R_{2}}\right)^{T M^{m} / 2}$

$$
+M^{m}\left(\frac{18 R_{1}}{M\left|y_{m}\right|}\right)^{T M^{m} / 2}\left(\frac{\left|y_{m}\right|}{2 \delta(M)}\right)^{T M^{m-1} / 2} \max _{\substack{\underline{h} \cdot \underline{y} \in Y(M) \\ 0 \leq k<T / 2}}\left|\frac{\widetilde{g}_{\underline{t}}^{(k)}(\underline{h} \cdot \underline{y})}{k !}\right|,
$$

where $\widetilde{g}_{\underline{t}}^{(k)}(z)=(\partial / \partial z)^{k} \widetilde{g}_{\underline{t}}(z)$. From hypothesis $\left(\mathrm{H}_{\mathrm{A}}\right)$ we have $\delta(M) \geq$ $\exp \left(-c_{0}^{\prime} M \log M\right)$, since $M \geq S_{0}$. We shall estimate $\left|\widetilde{g}_{\underline{t}}^{(k)}(\underline{h} \cdot \underline{y})\right|$. We infer from Lemma 1 and $t\left(H_{\alpha \underline{\beta} \underline{\underline{h}} \underline{\underline{t}}}\right) \leq c_{12} \Delta(S)$ that

$$
\left|\sum_{\alpha \leq D_{0}-1} \sum_{|\underline{\beta}| \leq D_{1}-1} \sum_{\underline{Z} \underline{\lambda} \in E} D^{\underline{i}} P_{\alpha \underline{\beta}} \underline{\lambda}(\underline{\tilde{\theta}}) H_{\alpha \underline{\beta} \underline{\lambda} \underline{h} \underline{\underline{t}}}(\underline{\theta})\right| \leq \exp \left(-\frac{c}{3} \varrho(S)\right)
$$

for all $\underline{h} \in \mathbb{N}^{m}(M)$ and all $\underline{t} \in \mathbb{N}^{n}$ with $|\underline{t}|<T$. Then we have the following equality analogous to (4):

$$
\begin{aligned}
& \text { (11) } Q(\underline{\theta})^{T+D_{0}+m D_{1} S}\left(\frac{u_{j_{\underline{h}}}^{\beta_{\underline{h}}}(\underline{\theta})}{\Theta_{j_{\underline{h}}}(\operatorname{Lie} \psi(\underline{h} \cdot \underline{y}))}\right)^{c_{8} D_{2}} \\
& \times D_{\underline{a}_{1}}^{t_{1}} \cdots D_{\underline{a}_{n}}^{t_{n}}\left(\frac{\widetilde{F}(\underline{z}+\operatorname{Lie} \varphi(\underline{h} \cdot \underline{y}))}{\Theta_{j_{0}}\left(\underline{z}^{\prime}\right)^{c_{8} D_{2}}}\right)_{\underline{z}=0}=\sum_{\substack{\alpha \leq D_{0}-1 \\
|\underline{\beta}| \leq D_{1}-1 \\
\underline{\underline{Z}} \underline{\underline{\lambda}} \in E}} D^{\underline{i}} P_{\alpha \underline{\beta} \underline{\underline{\lambda}}(\underline{\tilde{\theta}}) H_{\alpha \underline{\beta}} \underline{\lambda} \underline{\underline{h}} \underline{\underline{\theta}}(\underline{\theta})} .
\end{aligned}
$$

From the assumption in this case we have

$$
\left|u_{\underline{j_{\underline{h}}}}^{\beta_{\underline{h}}}(\underline{\theta})\right| \geq \exp \left(-\frac{c}{5} \frac{\varrho(S)}{D_{2}-1}\right) \quad \text { for } \underline{h} \in \mathbb{Z}^{m}(S) .
$$

Further, since $\Theta_{j_{h}}$ is of order $\leq 2$, we obtain $\left|\Theta_{j_{h}}(\operatorname{Lie} \psi(\underline{h} \cdot \underline{y}))\right| \leq \exp \left(c_{20} S^{2}\right)$, and finally $|Q(\underline{\theta})| \geq c_{21}>0$. Hence from (10) and (11) we have 


$$
\begin{aligned}
\mid D_{\underline{a}_{1}}^{t_{1}} \cdots D_{\underline{a}_{n}}^{t_{n}} & \left(\frac{\widetilde{F}(\underline{z}+\operatorname{Lie} \varphi(\underline{h} \cdot \underline{y}))}{\Theta_{j_{0}}\left(\underline{z}^{\prime}\right)^{c_{8} D_{2}}}\right)_{\underline{z}=0} \mid \\
& \leq \exp \left(-\frac{c}{3} \varrho(S)\right) \exp \left(\frac{c}{5} \varrho(S)\right) \exp \left(\left(c_{17}+c_{20}\right) \Delta(S)\right) \\
& \leq \exp \left(-\frac{c}{10} \varrho(S)\right)
\end{aligned}
$$

for all $\underline{h} \in \mathbb{N}^{m}(M)$ and all $\underline{t} \in \mathbb{N}^{n}$ with $|\underline{t}|<T$. We shall use the following identity:

$$
\begin{aligned}
& D_{\underline{a}_{1}}^{t_{1}} \cdots D_{\underline{a}_{n}}^{t_{n}} \widetilde{F}(\operatorname{Lie} \varphi(\underline{h} \cdot \underline{y})) \\
&=\sum_{\substack{0 \leq \tau_{i} \leq t_{i} \\
1 \leq i \leq n}}\left(\begin{array}{c}
t_{1} \\
\tau_{1}
\end{array}\right) \cdots\left(\begin{array}{c}
t_{n} \\
\tau_{n}
\end{array}\right) D_{\underline{a}_{1}}^{t_{1}-\tau_{1}} \cdots D_{\underline{a}_{n}}^{t_{n}-\tau_{n}}\left(\frac{\widetilde{F}(\underline{z}+\operatorname{Lie} \varphi(\underline{h} \cdot \underline{y}))}{\Theta_{j_{0}}\left(\underline{z}^{\prime}\right)^{c_{8} D_{2}}}\right)_{\underline{z}=0} \\
& \quad \times D_{\underline{\underline{x}}_{1}}^{\tau_{1}} \cdots D_{\underline{\underline{a}}_{n}}^{\tau_{n}}\left(\Theta_{j_{0}}\left(\underline{z}^{\prime}\right)^{c_{8} D_{2}}\right)_{\underline{z}^{\prime}=0} .
\end{aligned}
$$

Then (12) yields

$$
\left|D_{\underline{a}_{1}}^{t_{1}} \cdots D_{\underline{a}_{n}}^{t_{n}} \widetilde{F}(\operatorname{Lie} \varphi(\underline{h} \cdot \underline{y}))\right| \leq \exp \left(-\frac{c}{15} \varrho(S)\right)
$$

for all $\underline{h} \in \mathbb{N}^{m}(M)$ and all $\underline{t} \in \mathbb{N}^{n}$ with $|\underline{t}|<T$. From our assumption, $\operatorname{Lie} \varphi(\mathbb{C}) \subset W$, we have Lie $\varphi(z)=\ell_{1}(z) \underline{a}_{1}+\cdots+\ell_{n}(z) \underline{a}_{n}$ for $z \in \mathbb{C}$, where $\ell_{1}(z)=\ell_{1} z, \ldots, \ell_{n}(z)=\ell_{n} z$ for some complex numbers $\ell_{1}, \ldots, \ell_{n}$. Note that

$$
\widetilde{g}_{\underline{t}}^{(k)}(z)=\sum_{\substack{t_{1}^{\prime}+\cdots+t_{n}^{\prime}=k \\ t_{i}^{\prime} \geq 0}} \frac{k !}{t_{1}^{\prime} ! \cdots t_{n}^{\prime} !} \ell_{1}^{t_{1}^{\prime}} \cdots \ell_{n}^{t_{n}^{\prime}} D_{\underline{a}_{1}}^{t_{1}+t_{1}^{\prime}} \cdots D_{\underline{a}_{n}}^{t_{n}+t_{n}^{\prime}} \widetilde{F}(\operatorname{Lie} \varphi(z))
$$

for an integer $k \geq 0$. Hence

$$
\left|\widetilde{g}_{\underline{t}}^{(k)}(\underline{h} \cdot \underline{y})\right| \leq \exp \left(c_{21} T\right) \exp \left(-\frac{c}{15} \varrho(S)\right) \leq \exp \left(-\frac{c}{20} \varrho(S)\right)
$$

for all $\underline{h} \in \mathbb{N}^{m}(M)$, all $\underline{t} \in \mathbb{N}^{n}$ with $|\underline{t}|<T / 2$, and $0 \leq k \leq T / 2$. It follows easily that

$$
\left|\widetilde{g}_{\underline{t}}\right|_{R_{2}} \leq \exp \left(c_{22}\left(T \log D_{0}+T \log D_{1}+D_{0} \log R_{2}+D_{1} R_{2}+D_{2} R_{2}^{2}\right)\right),
$$

and taking into account (9) and (13), we have $\left|\widetilde{g}_{\underline{t}}\right|_{R_{1}} \leq \exp \left(-c_{23} \varrho(S)\right)$, since $T M^{m} \log S \gg \ll \varrho(S)$. Therefore

$$
\left|\widetilde{g}_{\underline{t}}(\underline{h} \cdot \underline{y})\right| \leq \exp \left(-c_{23} \varrho(S)\right)
$$

for $\underline{h} \in \mathbb{N}^{m}(S)$. From the properties of the theta function (cf. [20, lemme $2.2])$, we have $\max \left|\Theta_{i}(\operatorname{Lie} \psi(\underline{h} \cdot \underline{y}))\right| \geq \exp \left(-c_{24} S^{2}\right)$, and we also obtain $\max \left|u_{i}^{\beta_{\underline{h}}}(\underline{\theta})\right| \leq \exp \left(c_{24} S^{2}\right)$, and $|Q(\underline{\theta})|^{T+D_{0}+m D_{1} S} \leq \exp \left(c_{25} \Delta(S)\right)$. Thus 
we deduce from (8), (11), and (14) that

$$
\left|\sum_{\alpha \leq D_{0}-1} \sum_{|\underline{\beta}| \leq D_{1}-1} \sum_{\underline{Z} \underline{\underline{\lambda}} \in E} D^{\underline{i}} P_{\alpha \underline{\beta} \underline{\lambda}}(\underline{\tilde{\theta}}) H_{\alpha \underline{\beta} \underline{\lambda} \underline{h} \underline{\underline{t}}}(\underline{\theta})\right| \leq \exp \left(-c_{26} \varrho(S)\right)
$$

for all $\underline{h} \in \mathbb{N}^{m}(S)$ and all $\underline{t} \in \mathbb{N}^{n}$ with $|\underline{t}|<T / 2$. Finally, Lemma 1 shows that

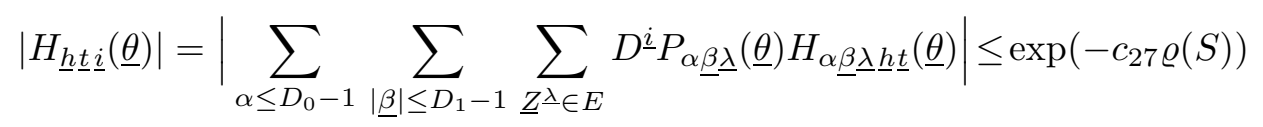

for all $\underline{h} \in \mathbb{N}^{m}(S)$ and all $\underline{t} \in \mathbb{N}^{n}$ with $|\underline{t}|<T / 2$.

This completes the proof of Lemma 4 .

\section{SteP 3: Philippon's zero estimate}

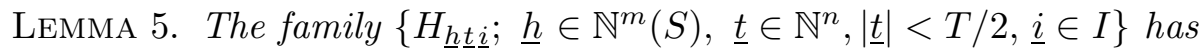
no common zeros in $B^{q}(\underline{\theta}, \exp (-c \varrho(S)))$.

Proof. The proof is by contradiction. Suppose that there exists a point $\underline{\tilde{\theta}}=\left(\widetilde{\theta}_{1}, \ldots, \widetilde{\theta}_{q}\right) \in B^{q}(\underline{\theta}, \exp (-c \varrho(S)))$ such that $H_{\underline{h} \underline{t} \underline{i}}(\underline{\widetilde{\theta}})=0$ for all $\underline{h} \in$ $\mathbb{N}^{m}(S)$, all $\underline{t} \in \mathbb{N}^{n}$ with $|\underline{t}|<T / 2$, and all $\underline{i} \in I$.

Let $\underline{\widetilde{a}}_{1}, \ldots, \underline{\widetilde{a}}_{n}$, and $\widetilde{W}$ be as in $\S 2$. We see that $\underline{\widetilde{a}}_{1}, \ldots, \underline{\widetilde{a}}_{n}$ are linearly independent over $\mathbb{C}$. Denote by $D_{\underline{\widetilde{a}}_{1}}, \ldots, D_{\underline{a}_{n}}$ the differential operators corresponding to $\underline{\widetilde{a}}_{1}, \ldots, \underline{\widetilde{a}}_{n}$ (cf. the beginning of this section). Let $\left(\widetilde{y}_{1}, \ldots, \widetilde{y}_{m-\ell}\right)$ be the element of $T_{G}(\mathbb{C})^{m-\ell}$ defined as above. Then by the same arguments as in the previous section, we have

$$
\begin{aligned}
& (Q(\underline{\widetilde{\theta}}))^{T+D_{0}+m D_{1} S}\left(\frac{u_{j_{\underline{h}}}^{\beta_{\underline{h}}}(\underline{\widetilde{\theta}})}{\Theta_{j_{\underline{h}}}\left(p_{2}(\underline{h} \cdot \widetilde{y})\right)}\right)^{c_{8} D_{2}} D_{\underline{\widetilde{a}}_{1}}^{t_{1}} \ldots D_{\underline{\widetilde{a}}_{n}}^{t_{n}}\left(\frac{(\widetilde{F}(\underline{z}+\underline{h} \cdot \widetilde{y})}{\Theta_{j_{0}}\left(\underline{z}^{\prime}\right)^{c_{8} D_{2}}}\right)_{\underline{z}=0} \\
& =\sum_{\substack{\alpha \leq D_{0}-1 \\
|\underline{\beta}| \leq D_{1}-1 \\
\underline{Z} \underline{\lambda}^{\underline{\lambda}} \in E}} D^{\underline{i}} P_{\alpha \underline{\beta} \underline{\lambda}}(\underline{\widetilde{\theta}}) H_{\alpha \underline{\beta} \underline{\lambda} \underline{h} \underline{t}}(\underline{\tilde{\theta}})=H_{\underline{h} \underline{t} \underline{i}}(\underline{\widetilde{\theta}})=0 .
\end{aligned}
$$

By the previous arguments, we then have $D_{\widetilde{a}_{1}}^{t_{1}} \cdots D_{\underline{a}_{n}}^{t_{n}} \widetilde{F}(\underline{h} \cdot \widetilde{y})=0$, since $Q(\underline{\widetilde{\theta}}) \neq 0$ and $u_{j_{\underline{h}}}^{\beta_{\underline{h}}}(\underline{\widetilde{\theta}}) \neq 0$. Hence $\widetilde{P}(X, \underline{Y}, \underline{Z})$ vanishes to order at least $[T / 2]$ along $\widetilde{W}$ on $\widetilde{\Gamma}(S)$. On the other hand, by our construction, $\widetilde{P}$ is not identically zero on $G$, and hence we see by Philippon's zero estimate [13, théorème 2.1] that there exists a connected algebraic subgroup $G^{\prime}$ of $G$ with $G^{\prime} \neq G$, incompletely defined by equations of multi-degree $\leq\left(\bar{D}_{0}, \bar{D}_{1}, 2 \bar{D}_{2}\right)$, such that

$$
\begin{aligned}
& \left(\begin{array}{c}
{\left[\frac{T-2}{2}\right]+\operatorname{codim}_{\widetilde{W}}\left(\widetilde{W} \cap T_{G^{\prime}}(\mathbb{C})\right)} \\
\operatorname{codim}_{\widetilde{W}}\left(\widetilde{W} \cap T_{G^{\prime}}(\mathbb{C})\right)
\end{array}\right) \operatorname{card}\left(\left(\widetilde{\Gamma}(S)+G^{\prime}\right) / G^{\prime}\right) \\
& \times H\left(G^{\prime} ; \bar{D}_{0}, \bar{D}_{1}, \bar{D}_{2}\right) \leq H\left(G ; \bar{D}_{0}, \bar{D}_{1}, 2 \bar{D}_{2}\right) .
\end{aligned}
$$


From our hypothesis $\left(\mathrm{H}_{\mathrm{B}}\right)$, we have

$$
\operatorname{codim}_{\widetilde{W}}\left(\widetilde{W} \cap T_{G^{\prime}}(\mathbb{C})\right) \geq \operatorname{codim}_{W}\left(W \cap T_{G^{\prime}}(\mathbb{C})\right) .
$$

As in Ably [1, p. 222], we deduce from hypothesis $\left(\mathrm{H}_{\mathrm{A}}\right)$ that

$$
\operatorname{card}\left(\left(\widetilde{\Gamma}(S)+G^{\prime}\right) / G^{\prime}\right) \geq \operatorname{card}\left(\left(\Gamma(S)+G^{\prime}\right) / G^{\prime}\right) .
$$

By the homogeneity of $H$, we infer from (15) that

$$
\begin{aligned}
\left.\left(\frac{T}{3}\right)^{\nu} \operatorname{card}\left(\left(\Gamma(S)+G^{\prime}\right) / G^{\prime}\right) \frac{H\left(G^{\prime} ; \bar{D}_{0} / B, \bar{D}_{1} / B, \bar{D}_{2} / B\right)}{H\left(G ; \bar{D}_{0} / B, \bar{D}_{1} / B,\right.}, \bar{D}_{2} / B\right) & \leq 2^{d_{2}} B^{\operatorname{dim} G / G^{\prime}} .
\end{aligned}
$$

Recalling the definition of $A\left(S, G^{\prime}\right)$ and $T$, and taking into account $A \leq$ $A\left(S, G^{\prime}\right)$ and $B \leq A$, we conclude from (16) that $c \leq 2^{d_{2}} 3^{\nu}$, which is impossible, since $c$ is sufficiently large. This completes the proof of Lemma 5 .

Proof of Proposition B (in Case 2). For $\underline{h} \in \mathbb{N}^{m}(S), \underline{t} \in \mathbb{N}^{n}$ with $|\underline{t}|<$ $T / 2$, and $\underline{i} \in I$, we put

$$
H_{\underline{h} \underline{\underline{t}} \underline{\underline{i}}}^{*}\left(\theta_{1}, \ldots, \theta_{q}\right):=r\left(H_{\underline{h} \underline{\underline{t}} \underline{\underline{t}}}\left(\theta_{1}, \ldots, \theta_{q}, X\right), R\left(\theta_{1}, \ldots, \theta_{q}, X\right)\right),
$$

where $r($,$) denotes Chudnovsky's semi-resultant. We see from [3, p. 207]$ and Lemma 4 that

$$
\left|H_{\underline{h} \underline{\underline{t}} \underline{i}}^{*}\left(\theta_{1}, \ldots, \theta_{q}\right)\right| \leq \exp \left(-c_{28} \varrho(S)\right), \quad t\left(H_{\underline{h} \underline{t} \underline{i}}^{*}\right) \leq c_{29} \Delta(S) .
$$

It follows from Lemma 5 that the family $\left\{H_{h \underline{t} \underline{i}}^{*} ; \underline{h} \in \mathbb{N}^{m}(S), \underline{t} \in \mathbb{N}^{n},|\underline{t}|<\right.$ $T / 2, \underline{i} \in I\}$ has no common zeros in $B^{q}(\underline{\theta}, \exp (-c \varrho(S)))$. If we denote by $\left\{P_{S, 1}, \ldots, P_{S, m(S)}\right\}$ the family of polynomials $\left\{H_{h t i}^{*} ; \underline{h} \in \mathbb{N}^{m}(S), \underline{t} \in \mathbb{N}^{n}\right.$, $|\underline{t}|<T / 2, \underline{i} \in I\}$ for $S \geq S_{0}$, and if we put $\mathcal{I}_{S}:=\left(\bar{P}_{S, j}\right)_{1 \leq j \leq m(S)}$, then the ideal $\mathcal{I}_{S}$ satisfies the conditions of Proposition B. This concludes the proof of Proposition B.

7. Proof of the Theorem. We shall show that the $\underline{\omega}$ of our Theorem satisfies the assumption of Proposition A. We may assume without loss of generality that $\theta_{j} \in K[\underline{\omega}]$ for all $j, 1 \leq j \leq q$. Put $F_{j}(\underline{\omega}):=\theta_{j}$, where $F_{j} \in K[\underline{Y}](1 \leq j \leq q)$, and put $Q_{S, i}(\underline{Y}):=P_{S, i}\left(F_{1}(\underline{Y}), \ldots, F_{q}(\underline{Y})\right)(1 \leq$ $i \leq m(S))$, where $P_{S, i}$ are the polynomials in $K\left[X_{1}, \ldots, X_{q}\right]$, constructed in Proposition B. It is clear from Proposition B that

$$
\left|Q_{S, i}(\underline{\omega})\right|=\left|P_{S, i}(\underline{\theta})\right| \leq \exp \left(-c_{30} \varrho(S)\right), \quad t\left(Q_{S, i}\right) \leq c_{31} t\left(P_{S, i}\right) \leq c_{32} \Delta(S) .
$$

On the other hand, it follows from Lemma 1 that for $j, 1 \leq j \leq q$,

$$
\left|F_{j}(\underline{\omega})-F_{j}(\underline{\widetilde{\omega}})\right| \leq \exp (-c \varrho(S)) \quad \text { whenever } \quad\|\underline{\widetilde{\omega}}-\underline{\omega}\| \leq \exp (-2 c \varrho(S)) .
$$


Putting $\underline{\widetilde{\theta}}=F_{j}(\underline{\widetilde{\omega}})$, we have $\|\underline{\theta}-\underline{\tilde{\theta}}\| \leq \exp (-c \varrho(S))$; and it follows from Proposition B that

$$
\max _{1 \leq i \leq m(S)}\left|P_{S, i}(\underline{\tilde{\theta}})\right|=\max _{1 \leq i \leq m(S)}\left|Q_{S, i}(\underline{\widetilde{\omega}})\right| \neq 0 .
$$

Hence for all $S \geq S_{0}$, we have:

(i) the family $\left(Q_{S, i}\right)_{1 \leq i \leq m(S)}$ has no common zeros in the ball with center $\underline{\omega}$ and radius $\exp (-2 c \varrho(S))$,

(ii) $\max _{1 \leq i \leq m(S)}\left|Q_{S, i}(\underline{\omega})\right| \leq \exp \left(-c_{30} \varrho(S)\right)$,

(iii) $\max _{1 \leq i \leq m(S)} t\left(Q_{S, i}\right) \leq c_{32} \Delta(S)$.

Now recall the definitions of $\varrho(S), \Delta(S)$, and $\kappa$. Put $N=c_{32} \Delta(S) ; \Delta(S)$ is a strictly increasing function, since $\mu^{\sharp}>0$. Let $\sigma$ be the inverse function of $c_{32} \Delta(S)$. Define a function $u: \mathbb{R}_{+} \rightarrow \mathbb{R}_{+}$by $u(N)=c_{30} \varrho(\sigma(N)) N^{-(k+1)}$. Since $\sigma(N)=S$, we have

$$
u(N)=c_{30} \varrho(S) N^{-(k+1)}=\frac{c_{30}}{c_{32}^{k+1}} S^{(\kappa-k-1) \mu^{\sharp}}(\log S)^{-k a} .
$$

For $\kappa \geq k+1$, the function $u$ is strictly increasing. In fact, if $\kappa>k+1$, this is obvious. If $\kappa=k+1$, we see from $(\mathcal{C} 1)$ that $-k a>0$. For all $N$ such that $\sigma(N) \geq S_{1}$, we put $G_{N, i}=Q_{\sigma(N), i}, 1 \leq i \leq m(\sigma(N))$. Then from properties (i), (ii), and (iii) above, for all $N$ satisfying $\sigma(N) \geq S_{1}$ we have

(i)' the family $\left(G_{N, i}\right)_{1 \leq i \leq m(\sigma(N))}$ has no common zeros in the ball with center $\underline{\omega}$ and radius $\exp \left(-\left(2 c / c_{30}\right) N^{k+1} u(N)\right)$,

(ii) ${ }^{\prime} \max _{1 \leq i \leq m(\sigma(N))}\left|G_{N, i}(\underline{\omega})\right| \leq \exp \left(-N^{k+1} u(N)\right)$,

(iii)' $\max _{1 \leq i \leq m(\sigma(N))} t\left(G_{N, i}\right) \leq N$.

Hence $\underline{\omega}$ satisfies the assumption of Proposition A. Now we have $u(N) \gg \ll$ $S^{(\kappa-k-1) \mu^{\sharp}}(\log S)^{-k a}$, where $N=c_{32} S^{\mu^{\sharp}}(\log S)^{a}$, and hence $u(N) \gg \ll$ $N^{\kappa-k-1}(\log N)^{-(\kappa-1) a}$. Note that

- if $\kappa=k+1$, the inverse function $v$ of $u$ satisfies $\log v(T) \gg \ll T^{-1 /(\kappa-1) a}$,

- if $\kappa>k+1$, then

$$
v(T) \gg \ll\left(\frac{T}{(\log T)^{-(\kappa-1) a}}\right)^{1 /(\kappa-k-1)} .
$$

Then Proposition A shows that there exists $c_{1}=c_{1}\left(G,[K: \mathbb{Q}], \phi, \chi, x_{1}, \ldots\right.$ $\left.\ldots, x_{d_{1}}, y_{1}, \ldots, y_{m}, k, L\right)>0$ such that

- if $\kappa=k+1$, the function $\Phi_{1}(T)=\exp \left(c_{1} T^{-1 /(\kappa-1) a}\right)$ is a measure of algebraic independence of $\underline{\omega}$ at dimension $k$,

- if $\kappa>k+1$, the function $\Phi_{2}(T)=c_{1}\left(T /(\log T)^{-(\kappa-1)(k+1) a / \kappa}\right)^{\kappa /(\kappa-k-1)}$ is a measure of algebraic independence of $\underline{\omega}$ at dimension $k$.

This completes the proof of the Theorem. 


\section{Proofs of corollaries}

\section{Exponential case}

Proof of Corollary 2. First, we note that $L$ is an arbitrary subfield of $\mathbb{C}$ and $a_{h p} \in L$ (for all $h, p$ ).

For $\sigma=1($ resp. $\sigma=2)$, we take $K=\mathbb{Q}, G=\mathbb{G}_{\mathrm{m}}^{d_{1}}\left(\right.$ resp. $\left.G=\mathbb{G}_{\mathrm{a}} \times \mathbb{G}_{\mathrm{m}}^{d_{1}}\right)$, $\underline{\omega}=\underline{\omega}^{(1)}=\left(a_{h p}, e^{x_{i} y_{j}} ; 1 \leq h \leq d_{1}, 1 \leq p \leq r, 1 \leq i \leq d_{1}, 1 \leq j \leq m\right)$ (resp. $\left.\underline{\omega}=\underline{\omega}^{(2)}=\left(a_{h p}, y_{j}, e^{x_{i} y_{j}} ; 0 \leq h \leq d_{1}, 0 \leq p \leq r, 1 \leq i \leq d_{1}, 1 \leq j \leq m\right)\right)$. We consider the one-parameter subgroup $\varphi: \mathbb{C} \rightarrow G(\mathbb{C})$ defined by $\varphi(z)=$ $\left(\exp \left(x_{1} z\right), \ldots, \exp \left(x_{d_{1}} z\right)\right)$ (resp. $\left.\varphi(z)=\left(z, \exp \left(x_{1} z\right), \ldots, \exp \left(x_{d_{1}} z\right)\right)\right)$. Put $Y=\mathbb{Z} y_{1}+\cdots+\mathbb{Z} y_{m}, \Gamma=\varphi(Y)$. Note that for both $\sigma=1$ and $\sigma=2$, we have $\operatorname{ker} \varphi=\{0\}$; hence $\ell=\operatorname{rank}_{\mathbb{Z}}(Y \cap \operatorname{ker} \varphi)=0$.

We put $\underline{a}_{p}=\left(a_{1 p}, \ldots, a_{d_{1} p}\right)(1 \leq p \leq r)\left(\operatorname{resp} . \underline{a}_{p}=\left(a_{0 p}, \ldots, a_{d_{1} p}\right)\right.$ $(0 \leq p \leq r))$ and $W^{(1)}=\mathbb{C} \underline{a}_{1}+\cdots+\mathbb{C} \underline{a}_{r}\left(\operatorname{resp} . W^{(2)}=\mathbb{C} \underline{a}_{0}+\cdots+\mathbb{C} \underline{a}_{r}\right)$. For brevity, we put $W=W^{(1)}$ or $W^{(2)}$. From $\left(x_{1}, \ldots, x_{d_{1}}\right)=x_{1} \underline{a}_{1}+\cdots+x_{r} \underline{a}_{r}$ (resp. $\left.\left(x_{0}, \ldots, x_{d_{1}}\right)=x_{0} \underline{a}_{0}+\cdots+x_{r} \underline{a}_{r}\right)$, we have Lie $\varphi(\mathbb{C}) \subset W$. First, for any algebraic subgroup $G^{\prime} \subsetneq G$, we note that $\operatorname{Lie} \varphi(\mathbb{C}) \cap T_{G^{\prime}}(\mathbb{C})=\{0\}$. In fact, otherwise we have $\operatorname{Lie} \varphi(\mathbb{C}) \subset T_{G^{\prime}}(\mathbb{C})$, because $\operatorname{dim}_{\mathbb{C}} \operatorname{Lie} \varphi(\mathbb{C})=1$, which contradicts the fact that $\varphi(\mathbb{C})$ is Zariski-dense in $G(\mathbb{C})$ and $G^{\prime}(\mathbb{C}) \subsetneq G(\mathbb{C})$. Hence for any algebraic subgroup $G^{\prime} \subsetneq G$, we also deduce that $\Gamma \cap G^{\prime}=\{0\}$. Next, we note for any algebraic subgroup $G^{\prime} \subsetneq G$ that $W \cap T_{G^{\prime}}(\mathbb{C})=\{0\}$, for otherwise we have $W \cap T_{G^{\prime}}(\mathbb{C}) \neq\{0\}$ and $W \cap T_{G^{\prime}}(\mathbb{C}) \neq W$. On the other hand, since Lie $\varphi(\mathbb{C}) \cap\left(W \cap T_{G^{\prime}}(\mathbb{C})\right)=\operatorname{Lie} \varphi(\mathbb{C}) \cap T_{G^{\prime}}(\mathbb{C})=\{0\}$, Lie $\varphi(\mathbb{C})$ is contained in the orthogonal complement of $W \cap T_{G^{\prime}}(\mathbb{C})$ in $W$, which contradicts the choice of $W$. Hence we see that $\mu_{1}^{\sharp}$ attains its minimum when $G^{\prime}=\{0\}$, and so

$$
\mu_{1}^{\sharp}=\frac{m+d_{1}}{d_{1}-r} \quad \text { and } \quad \kappa_{1}=\frac{\left(d_{1}-r\right) m}{m+d_{1}}+1 .
$$

Hypothesis $\left(\mathrm{H}_{\mathrm{A}}\right)$ is a consequence of hypothesis $\left(\mathrm{H}_{1}\right)$ and the description of the connected algebraic subgroups of $\mathbb{G}_{\mathrm{m}}^{d_{1}}$ (resp. of $\mathbb{G}_{\mathrm{a}} \times \mathbb{G}_{\mathrm{m}}^{d_{1}}$ ) (see [22] and also [4]). We shall prove that $\left(\mathrm{H}_{\mathrm{B}}\right)$ follows from hypothesis $\mathrm{H}\left(L, \mathbb{Z} ;\left(\mu_{1}^{\sharp}+m\right) / d_{1}\left(\mu_{1}^{\sharp}-1\right)\right)$.

Lemma 6. $\mathrm{H}\left(L, \mathbb{Z} ;\left(\mu_{1}^{\sharp}+m\right) /\left(\mu_{1}^{\sharp}-1\right) d_{1}\right) \Rightarrow\left(\mathrm{H}_{\mathrm{B}}\right)$.

Proof. Here we shall only give the proof in the case $G=\mathbb{G}_{\mathrm{m}}^{d_{1}}$. Note that $W /\left(W \cap T_{G^{\prime}}(\mathbb{C})\right) \cong\left(W+T_{G^{\prime}}(\mathbb{C})\right) / T_{G^{\prime}}(\mathbb{C})$. Put $\nu=\operatorname{dim} W /\left(W \cap T_{G^{\prime}}(\mathbb{C})\right)$. We suppose that $\underline{a}_{j_{1}}+T_{G^{\prime}}(\mathbb{C}), \ldots, \underline{a}_{j_{\nu}}+T_{G^{\prime}}(\mathbb{C})$ (say) are linearly independent over $\mathbb{C}$ in the vector space $T_{G}(\mathbb{C}) / T_{G^{\prime}}(\mathbb{C})$. Then we shall show under the hypothesis $\mathrm{H}\left(L, \mathbb{Z} ;\left(\mu_{1}^{\sharp}+m\right) /\left(\mu_{1}^{\sharp}-1\right) d_{1}\right)$ that $\underline{\tilde{a}}_{j_{1}}+T_{G^{\prime}}(\mathbb{C}), \ldots, \underline{\tilde{a}}_{j_{\nu}}+T_{G^{\prime}}(\mathbb{C})$ are linearly independent over $\mathbb{C}$, which yields $\left(\mathrm{H}_{\mathrm{B}}\right)$. The proof is by contradiction. Assume that $\underline{\widetilde{a}}_{j_{1}}+T_{G^{\prime}}(\mathbb{C}), \ldots, \underline{\widetilde{a}}_{j_{\nu}}+T_{G^{\prime}}(\mathbb{C})$ are linearly dependent 
over $\mathbb{C}$, and hence there exist $\nu$ complex numbers $e_{1}, \ldots, e_{\nu}$ not all zero such that $e_{1} \underline{\tilde{a}}_{j_{1}}+\cdots+e_{\nu} \underline{\widetilde{a}}_{j_{\nu}} \in T_{G^{\prime}}(\mathbb{C})$. Since $\underline{\tilde{a}}_{j_{s}}=\left(\widetilde{a}_{1 j_{s}}, \ldots, \widetilde{a}_{d_{1} j_{s}}\right)(1 \leq s \leq \nu)$, we have

$$
\left(e_{1} \widetilde{a}_{1 j_{1}}+\cdots+e_{\nu} \widetilde{a}_{1 j_{\nu}}, \ldots, e_{1} \widetilde{a}_{d_{1} j_{1}}+\cdots+e_{\nu} \widetilde{a}_{d_{1} j_{\nu}}\right) \in T_{G^{\prime}}(\mathbb{C}) .
$$

Then by Bertrand's theorem [6, Annexe], there exist $\delta\left(=\operatorname{dim} G / G^{\prime}\right)$ linearly independent integer points $\underline{\lambda}^{(1)}, \ldots, \underline{\lambda}^{(\delta)} \in \mathbb{Z}^{d_{1}}$ such that

$$
\begin{gathered}
\lambda_{1}^{(\varrho)}\left(e_{1} \widetilde{a}_{1 j_{1}}+\cdots+e_{\nu} \widetilde{a}_{1 j_{\nu}}\right)+\cdots+\lambda_{d_{1}}^{(\varrho)}\left(e_{1} \widetilde{a}_{d_{1} j_{1}}+\cdots+e_{\nu} \widetilde{a}_{d_{1} j_{\nu}}\right)=2 m_{\varrho} \pi i \\
(1 \leq \varrho \leq \delta), \\
\prod_{\varrho=1}^{\delta}\left\|\underline{\lambda}^{(\varrho)}\right\| \leq c\left(\delta, d_{1}\right) D_{1}^{\delta},
\end{gathered}
$$

where $\underline{\lambda}^{(\varrho)}=\left(\lambda_{1}^{(\varrho)}, \ldots, \lambda_{d_{1}}^{(\varrho)}\right), m_{\varrho} \in \mathbb{Z}(1 \leq \varrho \leq \delta)$ and $c\left(\delta, d_{1}\right)$ is a positive constant depending only on $\delta$ and $d_{1}$. We rewrite this system of linear equations as follows:

$$
\begin{gathered}
\widetilde{p}_{11} e_{1}+\cdots+\widetilde{p}_{1 \nu} e_{\nu}=2 m_{1} \pi i, \\
\ldots \ldots+\cdots \cdots+\cdots \cdots+\cdots \cdots+\widetilde{p}_{\delta \nu} e_{\nu}=2 m_{\delta} \pi i \\
\widetilde{p}_{\delta 1} e_{1}+\cdots+
\end{gathered}
$$

where for simplicity, we put

$$
\begin{aligned}
& \widetilde{p}_{\varrho 1}=\lambda_{1}^{(\varrho)} \widetilde{a}_{1 j_{1}}+\cdots+\lambda_{d_{1}}^{(\varrho)} \widetilde{a}_{d_{1} j_{1}}, \ldots, \\
& \widetilde{p}_{\varrho \nu}=\lambda_{1}^{(\varrho)} \widetilde{a}_{1 j_{\nu}}+\cdots+\lambda_{d_{1}}^{(\varrho)} \widetilde{a}_{d_{1} j_{\nu}} \quad(1 \leq \varrho \leq \delta) .
\end{aligned}
$$

Denote by $p_{\varrho s}$ the linear forms $\widetilde{p}_{\varrho s}$ of $\widetilde{a}_{h j_{s}}$ replaced by $a_{h j_{s}}(1 \leq \varrho \leq \delta, 1 \leq$ $\left.h \leq d_{1}, 1 \leq s \leq \nu\right)$. Let $P=\left(p_{\varrho s}\right)_{1 \leq \varrho \leq \delta, 1 \leq s \leq \nu}$ denote a $\delta \times \nu$ matrix.

First, we suppose that $\underline{m}=\left(m_{1}, \ldots, m_{\delta}\right) \neq(0, \ldots, 0)$. By the well known result of linear algebra, if rank $P<\nu$, then the system of linear equations

$$
\begin{gathered}
p_{11} z_{1}+\cdots+p_{1 \nu} z_{\nu}=2 m_{1} \pi i, \\
\ldots \ldots+\cdots \cdots+\cdots \cdots+\cdots \cdots+p_{\delta \nu} z_{\nu}=2 m_{\delta} \pi i \\
p_{\delta 1} z_{1}+\cdots+
\end{gathered}
$$

has a nontrivial solution. If $\operatorname{rank} P=\nu$, we shall consider two cases.

CASE (i): $\nu=\delta$. Then (18) has a unique (nontrivial) solution.

CASE (ii): $\nu<\delta$. Then (17) has a nontrivial solution $\left(e_{1}, \ldots, e_{\nu}\right) \neq$ $(0, \ldots, 0)$. This means that

$$
\operatorname{rank}\left(\begin{array}{ccc}
\widetilde{p}_{11} & \cdots & \widetilde{p}_{1 \nu} \\
\vdots & \ddots & \vdots \\
\widetilde{p}_{\delta 1} & \cdots & \widetilde{p}_{\delta \nu}
\end{array}\right)=\operatorname{rank}\left(\begin{array}{cccc}
\widetilde{p}_{11} & \cdots & \widetilde{p}_{1 \nu} & m_{1} \\
\vdots & \ddots & \vdots & \vdots \\
\widetilde{p}_{\delta 1} & \cdots & \widetilde{p}_{\delta \nu} & m_{\delta}
\end{array}\right) .
$$


Thus for any $\eta_{1}, \ldots, \eta_{\nu+1}$ with $1 \leq \eta_{1}<\cdots<\eta_{\nu+1} \leq \delta$, we have

$$
\left|\begin{array}{cccc}
\widetilde{p}_{\eta_{1}, 1} & \ldots & \widetilde{p}_{\eta_{1}, \nu} & m_{\eta_{1}} \\
\vdots & \ddots & \vdots & \vdots \\
\widetilde{p}_{\eta_{\nu+1}, 1} & \ldots & \widetilde{p}_{\eta_{\nu+1}, \nu} & m_{\eta_{\nu+1}}
\end{array}\right|=0 .
$$

Then from this equation and an elementary computation, we have

$$
\begin{aligned}
\left|\begin{array}{cccc}
p_{\eta_{1}, 1} & \cdots & p_{\eta_{1}, \nu} & m_{\eta_{1}} \\
\vdots & \ddots & \vdots & \vdots \\
p_{\eta_{\nu+1}, 1} & \ldots & p_{\eta_{\nu+1}, \nu} & m_{\eta_{\nu+1}}
\end{array}\right|=\left|\begin{array}{ccccc}
p_{\eta_{1}, 1}-\widetilde{p}_{\eta_{1}, 1} & p_{\eta_{1}, 2} & \cdots & m_{\eta_{1}} \\
\vdots & \vdots & \ddots & \vdots \\
p_{\eta_{\nu+1}, 1}-\widetilde{p}_{\eta_{\nu+1}, 1} & p_{\eta_{\nu+1}, 2} & \cdots & m_{\eta_{\nu+1}}
\end{array}\right| \\
\\
+\cdots+\left|\begin{array}{cccccc}
\widetilde{p}_{\eta_{1}, 1} & \cdots & \widetilde{p}_{\eta_{1}, \nu-1} & p_{\eta_{1}, \nu}-\widetilde{p}_{\eta_{1}, \nu} & m_{\eta_{1}} \\
\vdots & \ddots & \vdots & \vdots & & \vdots \\
\widetilde{p}_{\eta_{\nu+1}, 1} & \cdots & \widetilde{p}_{\eta_{\nu+1}, \nu-1} & p_{\eta_{\nu+1}, \nu}-\widetilde{p}_{\eta_{\nu+1}, \nu} & m_{\eta_{\nu+1}}
\end{array}\right| .
\end{aligned}
$$

For all $t(1 \leq t \leq \nu+1)$ and for all $s(1 \leq s \leq \nu)$, we easily obtain

$$
\begin{gathered}
\left|p_{\eta_{t}, s}-\widetilde{p}_{\eta_{t}, s}\right| \leq c_{33}\left\|\underline{\lambda}^{\left(\eta_{t}\right)}\right\| \max _{\substack{1 \leq h \leq d_{1} \\
1 \leq s \leq \nu}}\left|a_{h j_{s}}-\widetilde{a}_{h j_{s}}\right| \leq\left\|\underline{\lambda}^{\left(\eta_{t}\right)}\right\| \exp \left(-\frac{c}{3} \varrho(S)\right), \\
\left|p_{\eta_{t}, s}\right|,\left|\widetilde{p}_{\eta_{t}, s}\right| \leq c_{34}\left\|\underline{\lambda}^{\left(\eta_{t}\right)}\right\|, \quad\left|m_{\eta_{t}}\right| \leq c_{34}\left\|\underline{\lambda}^{\left(\eta_{t}\right)}\right\| .
\end{gathered}
$$

Hence we have an upper bound

$$
\begin{gathered}
\text { (19) } \left.\operatorname{abs}\left(\mid \begin{array}{cccc}
p_{\eta_{1}, 1} & \cdots & p_{\eta_{1}, \nu} & m_{\eta_{1}} \\
\vdots & \ddots & & \vdots \\
p_{\eta_{\nu+1}, 1} & \cdots & p_{\eta_{\nu+1}, \nu} & m_{\eta_{\nu+1}}
\end{array}\right)\right) \\
\leq c_{35}\left\|\underline{\lambda}^{\left(\eta_{1}\right)}\right\| \cdots\left\|\underline{\lambda}^{\left(\eta_{\nu+1}\right)}\right\| \exp \left(-\frac{c}{3} \varrho(S)\right) \\
\leq c_{36} D_{1}^{d_{1}} \exp \left(-\frac{c}{3} \varrho(S)\right) \leq \exp \left(-\frac{c}{4} \varrho(S)\right) .
\end{gathered}
$$

Next, we shall find a lower bound for the above determinant. We use expansion along the $(\nu+1)$ th row:

$$
\left|\begin{array}{ccc}
p_{\eta_{1}, 1} & \cdots & p_{\eta_{\nu+1}, 1} \\
\vdots & \ddots & \vdots \\
p_{\eta_{1}, \nu} & \cdots & p_{\eta_{\nu+1}, \nu} \\
m_{\eta_{1}} & \cdots & m_{\eta_{\nu+1}}
\end{array}\right|=m_{\eta_{1}} A_{\eta_{1}}+\cdots+m_{\eta_{\nu+1}} A_{\eta_{\nu+1}}
$$


where $A_{\eta_{t}}$ is the cofactor of $m_{\eta_{t}}(1 \leq t \leq \nu+1)$. Hence we have

$$
\begin{aligned}
A_{\eta_{t}}= & \pm \sum_{i_{1}, \ldots, i_{\nu+1}} \lambda_{i_{1}}^{\left(\eta_{1}\right)} \cdots \lambda_{i_{t-1}}^{\left(\eta_{t-1}\right)} \lambda_{i_{t+1}}^{\left(\eta_{t+1}\right)} \cdots \lambda_{i_{\nu+1}}^{\left(\eta_{\nu+1}\right)} \\
& \times\left|\begin{array}{cccccc}
a_{i_{1} j_{1}} & \cdots & a_{i_{t-1} j_{1}} & a_{i_{t+1} j_{1}} & \cdots & a_{i_{\nu+1} j_{1}} \\
\vdots & \ddots & \vdots & \vdots & \ddots & \vdots \\
a_{i_{1} j_{\nu}} & \cdots & a_{i_{t-1} j_{\nu}} & a_{i_{t+1} j_{\nu}} & \cdots & a_{i_{\nu+1} j_{\nu}}
\end{array}\right|
\end{aligned}
$$

It follows easily that

$$
\left|m_{\eta_{t}} \lambda_{i_{1}}^{\left(\eta_{1}\right)} \cdots \lambda_{i_{t-1}}^{\left(\eta_{t-1}\right)} \lambda_{i_{t+1}}^{\left(\eta_{t+1}\right)} \cdots \lambda_{i_{\nu+1}}^{\left(\eta_{\nu+1}\right)}\right| \leq\left\|\underline{\lambda}^{\left(\eta_{1}\right)}\right\| \cdots\left\|\underline{\lambda}^{\left(\eta_{\nu+1}\right)}\right\| \leq c(\delta, n) D_{1}^{d_{1}} .
$$

Thus from hypothesis $\mathrm{H}\left(L, \mathbb{Z} ;\left(\mu_{1}^{\sharp}+m\right) /\left(\mu_{1}^{\sharp}-1\right) d_{1}\right)$, we have either

or

$$
\left|\begin{array}{cccc}
p_{\eta_{1}, 1} & \cdots & p_{\eta_{1}, \nu} & m_{\eta_{1}} \\
\vdots & \ddots & \vdots & \vdots \\
p_{\eta_{\nu+1}, 1} & \cdots & p_{\eta_{\nu+1}, \nu} & m_{\eta_{\nu+1}}
\end{array}\right|=0
$$

$$
\operatorname{abs}\left(\left|\begin{array}{cccc}
p_{\eta_{1}, 1} & \cdots & p_{\eta_{1}, \nu} & m_{\eta_{1}} \\
\vdots & \ddots & \vdots & \vdots \\
p_{\eta_{\nu+1}, 1} & \cdots & p_{\eta_{\nu+1}, \nu} & m_{\eta_{\nu+1}}
\end{array}\right|\right) \geq \exp \left(-c_{37} D_{1}^{d_{1} \alpha}\right),
$$

where $\alpha=\left(\mu_{1}^{\#}+m\right) /\left(\mu_{1}^{\#}-1\right) d_{1}$; but the latter contradicts (19), since $a<0$; and hence $\operatorname{rank} P=\operatorname{rank}\left(P,{ }^{t} \underline{m}\right)=\nu$, where ${ }^{t} \underline{m}$ denotes the transpose of $\underline{m}$. Thus (18) has a nontrivial solution.

In the case of $\left(m_{1}, \ldots, m_{\delta}\right)=(0, \ldots, 0)$, it is clear that $\operatorname{rank} \widetilde{P}<\nu$. Then using the same arguments as above, it follows from hypothesis $\mathrm{H}(L, \mathbb{Z}$; $\left.\mu_{1}^{\sharp} /\left(\mu_{1}^{\sharp}-1\right) d_{1}\right)$ that $\operatorname{rank} P<\nu$, which will be excluded.

Thus we have shown that (18) always has a nontrivial solution $\left(f_{1}, \ldots, f_{\nu}\right)$ (say). Now we see from (18) that

$$
\begin{array}{r}
f_{1}\left(\lambda_{1}^{(\varrho)} a_{1 j_{1}}+\cdots+\lambda_{d_{1}}^{(\varrho)} a_{d_{1} j_{1}}\right)+\cdots+f_{\nu}\left(\lambda_{1}^{(\varrho)} a_{1 j_{\nu}}+\cdots+\lambda_{d_{1}}^{(\varrho)} a_{d_{1} j_{\nu}}\right) \\
\left(1 \leq 2 m_{\varrho} \pi i\right. \\
(1 \leq \varrho \leq \delta) .
\end{array}
$$

We rewrite this equation as follows:

$$
\begin{array}{r}
\lambda_{1}^{(\varrho)}\left(f_{1} a_{1 j_{1}}+\cdots+f_{\nu} a_{1 j_{\nu}}\right)+\cdots+\lambda_{d_{1}}^{(\varrho)}\left(f_{1} a_{d_{1} j_{1}}+\cdots+f_{\nu} a_{d_{1} j_{\nu}}\right)=2 m_{\varrho} \pi i \\
\quad(1 \leq \varrho \leq \delta) .
\end{array}
$$

Hence using Bertrand's theorem [6, Annexe] again, we have $f_{1} \underline{a}_{j_{1}}+\cdots$ $\cdots+f_{\nu} \underline{a}_{j_{\nu}} \in T_{G^{\prime}}(\mathbb{C})$. This means that $\underline{a}_{j_{1}}+T_{G^{\prime}}(\mathbb{C}), \ldots, \underline{a}_{j_{\nu}}+T_{G^{\prime}}(\mathbb{C})$ are $\mathbb{C}$-linearly dependent in the vector space $T_{G}(\mathbb{C}) / T_{G^{\prime}}(\mathbb{C})$, which contradicts our choice of $\underline{a}_{j_{1}}, \ldots, \underline{a}_{j_{\nu}}$. Therefore $\operatorname{codim}_{W}\left(W \cap T_{G^{\prime}}(\mathbb{C})\right) \leq$ $\operatorname{codim}_{\widetilde{W}}\left(\widetilde{W} \cap T_{G^{\prime}}(\mathbb{C})\right)$.

This completes the proof of Lemma 6 . 
End of proof of Corollary 2. Since hypotheses $\left(\mathrm{H}_{\mathrm{A}}\right)$ and $\left(\mathrm{H}_{\mathrm{B}}\right)$ are satisfied, Corollary 2 follows immediately from our Theorem.

Proof of Corollary 4. Take $y_{1}=1, y_{2}=\beta, \ldots, y_{5}=\beta^{4} ; x_{1}=\log \alpha, x_{2}=$ $\beta \log \alpha, \ldots, x_{5}=\beta^{4} \log \alpha ; L=\mathbb{Q}(\beta)$ in Corollary 2 . Then the hypotheses of Corollary 2 are clearly satisfied. Thus the result follows from Corollary 3 by putting $m=d_{1}=5$, and $r=1$.

Elliptic case. Let $E=E(\mathbb{C})$ be the elliptic curve associated to $\wp, \Omega$ the lattice of periods of $\wp$, and $K=\mathbb{Q}\left(g_{2}, g_{3}\right)$, where $g_{2}$ and $g_{3}$ are the algebraic invariants of $\wp$. Let $\omega_{1}, \omega_{2}$ denote a fixed basis for $\Omega$, and hence $\Omega=$ $\left\{m_{1} \omega_{1}+m_{2} \omega_{2} ; m_{1}, m_{2} \in \mathbb{Z}\right\}$. Put $\tau=\omega_{2} / \omega_{1}$. The exponential map of $E$ is given by $\exp _{E}: \mathbb{C} \rightarrow E(\mathbb{C}) \subset \mathbb{P}_{2}(\mathbb{C})$,

$$
z \mapsto \begin{cases}\left(1, \wp(z), \wp^{\prime}(z)\right) & \text { if } z \notin \Omega, \\ (0,0,1) & \text { if } z \in \Omega .\end{cases}
$$

Proof of Corollary 5. If $\sigma=3$ (resp. $\sigma=4$ ), we take $G=E^{d_{1}}$ (resp. $G=$ $\left.\mathbb{G}_{\mathrm{a}} \times E^{d_{1}}\right)$. We consider the one-parameter subgroup $\varphi: \mathbb{C} \rightarrow G(\mathbb{C})$ defined by $\varphi(z)=\left(\exp _{E}\left(x_{1} z\right), \ldots, \exp _{E}\left(x_{d_{1}} z\right)\right)\left(\operatorname{resp} . \varphi(z)=\left(z, \exp _{E}\left(x_{1} z\right), \ldots\right.\right.$ $\left.\left.\ldots, \exp _{E}\left(x_{d_{1}} z\right)\right)\right)$.

CASE $1: \mathbb{F}=\mathbb{Q}$. We take $Y=\mathbb{Z} y_{1}+\cdots+\mathbb{Z} y_{m}$. If $\sigma=3$ (resp. $\left.\sigma=4\right)$, we put $\underline{\omega}=\left(a_{h p}, 1, \wp\left(x_{i} y_{j}\right), \wp^{\prime}\left(x_{i} y_{j}\right) ; 1 \leq h \leq d_{1}, 1 \leq p \leq r, 1 \leq i \leq d_{1}, 1 \leq\right.$ $\left.j \leq m, x_{i} y_{j} \notin \Omega\right)\left(\operatorname{resp} . \underline{\omega}=\left(a_{h p}, y_{j}, 1, \wp\left(x_{i} y_{j}\right), \wp^{\prime}\left(x_{i} y_{j}\right) ; 0 \leq h \leq d_{1}, 0 \leq\right.\right.$ $\left.\left.p \leq r, 1 \leq i \leq d_{1}, 1 \leq j \leq m, x_{i} y_{j} \notin \Omega\right)\right)$.

CASE $2: \mathbb{F} \neq \mathbb{Q}, \mathbb{F}=\mathbb{Q}(\tau)$, where $\tau$ is some quadratic irrational number. We take $Y=\mathbb{Z} y_{1}+\cdots+\mathbb{Z} y_{m}+\mathbb{Z} \tau y_{1}+\cdots+\mathbb{Z} \tau y_{m}$ and $\underline{\omega}=\left(a_{h p}, 1, \wp\left(x_{i} y_{j}\right)\right.$, $\wp^{\prime}\left(x_{i} y_{j}\right), 1, \wp\left(x_{i} y_{j} \tau\right), \wp^{\prime}\left(x_{i} y_{j} \tau\right) ; 1 \leq h \leq d_{1}, 1 \leq p \leq r, 1 \leq i \leq d_{1}, 1 \leq j \leq m$, $\left.x_{i} y_{j} \notin \Omega\right)$ (resp. $\underline{\omega}=\left(a_{h p}, y_{j}, 1, \wp\left(x_{i} y_{j}\right), \wp^{\prime}\left(x_{i} y_{j}\right), 1, \wp\left(x_{i} y_{j} \tau\right), \wp^{\prime}\left(x_{i} y_{j} \tau\right)\right.$; $\left.\left.0 \leq h \leq d_{1}, 0 \leq p \leq r, 1 \leq i \leq d_{1}, 1 \leq j \leq m, x_{i} y_{j} \notin \Omega\right)\right)$. Recall that $\underline{\omega}^{(3)}=\left(a_{h p}, \wp\left(x_{i} y_{j}\right) ; 1 \leq h \leq d_{1}, 1 \leq p \leq r, 1 \leq i \leq d_{1}, 1 \leq j \leq m, x_{i} y_{j} \notin\right.$ $\Omega)$ and $\underline{\omega}^{(4)}=\left(a_{h p}, y_{j}, \wp\left(x_{i} y_{j}\right) ; 0 \leq h \leq d_{1}, 0 \leq p \leq r, 1 \leq i \leq d_{1}, 1 \leq j \leq m\right.$, $\left.x_{i} y_{j} \notin \Omega\right)$.

We put $\underline{a}_{p}=\left(a_{1 p}, \ldots, a_{d_{1} p}\right)(1 \leq p \leq r)\left(\right.$ resp. $\underline{a}_{p}=\left(a_{0 p}, \ldots, a_{d_{1} p}\right)$ $(0 \leq p \leq r))$ and $W^{(3)}=\mathbb{C} \underline{a}_{1}+\cdots+\mathbb{C} \underline{a}_{r}\left(\operatorname{resp} . W^{(4)}=\mathbb{C} \underline{a}_{0}+\cdots+\mathbb{C} \underline{a}_{r}\right)$. From the same arguments as in the exponential case, we see that $\mu_{2}^{\sharp}$ attains its minimum when $G^{\prime}=\{0\}$, and hence

$\mu_{2}^{\sharp}=\left([\mathbb{F}: \mathbb{Q}] m+2 d_{1}\right) /\left(d_{1}-r\right), \quad \kappa_{2}=\left([\mathbb{F}: \mathbb{Q}]\left(d_{1}-r\right) m\right) /\left([\mathbb{F}: \mathbb{Q}] m+2 d_{1}\right)+1$.

$\left(\mathrm{H}_{\mathrm{A}}\right)$ is a consequence of hypothesis $\left(\mathrm{H}_{2}\right)$ and the description of the connected algebraic subgroups of $E^{d_{1}}$ (resp. $\mathbb{G}_{\mathrm{a}} \times E^{d_{1}}$ ) with the aid of the 
effective Kolchin theorem (cf. [10]). Since $\left(\mathrm{H}_{\mathrm{B}}\right)$ is derived from hypothesis

$$
\mathrm{H}\left(L, \mathbb{Z}+\mathbb{Z} \tau ; \frac{[\mathbb{F}: \mathbb{Q}] m+\mu_{2}^{\sharp}}{2 d_{1}\left(1+\log d_{1}\right)\left(\mu_{2}^{\sharp}-2\right)}\right)
$$

by the arguments similar to those of the exponential case, we shall omit its proof. Finally, since $K(\underline{\omega})$ is algebraic over $K\left(\underline{\omega}^{(\sigma)}\right)(\sigma=3,4)$, Corollary 5 is easily deduced from our Theorem and a remark in [1, p. 225].

Proof of Corollary 7. We shall deduce this result from Corollary 6, in the case $\sigma=4$. We take $y_{j}=\beta^{j-1}(j=1, \ldots, \delta), x_{i}=\beta^{i-1} u(i=1, \ldots, \delta)$. We put $L=\mathbb{Q}(\beta)$. Then it is clear that $\{1, u\}$ are linearly independent over $L$ and that $1, u, \beta u, \ldots, \beta^{\delta-1} u$ are linearly dependent on $\{1, u\}$ over $L$. We put

$$
\underline{a}_{0}=\underbrace{(1,0, \ldots, 0)}_{\delta+1}, \quad \underline{a}_{1}=\underbrace{\left(0,1, \beta, \ldots, \beta^{\delta-1}\right)}_{\delta+1}
$$

and $W=\mathbb{C} \underline{a}_{0}+\mathbb{C} \underline{a}_{1} ;$ hence we have $\operatorname{dim}_{\mathbb{C}} W=2$ and $r=1$.

It is easily checked that hypothesis $\left(\mathrm{H}_{2}\right)$ (and hence $\left(\mathrm{H}_{\mathrm{A}}\right)$ ) is satisfied. Now we shall show that hypothesis $\left(\mathrm{H}_{\mathrm{B}}\right)$ is always satisfied.

Lemma 7. Under the assumption of Corollary $7,\left(\mathrm{H}_{\mathrm{B}}\right)$ is true.

Proof. The proof is similar to that of the exponential case.

We consider $G=\mathbb{G}_{\mathrm{a}} \times E^{\delta}$. Any connected algebraic subgroup $G^{\prime}$ of $G$ with $G^{\prime} \neq G$ has the form $G^{\prime}=G_{0}^{\prime} \times G_{2}^{\prime}$, where $G_{0}^{\prime}=\{0\}$ or $\mathbb{G}_{\mathrm{a}}$, and $G_{2}^{\prime}$ is a connected algebraic subgroup of $E^{\delta}$. Since $W \cap T_{G^{\prime}}(\mathbb{C})=\{0\}$, it is obvious that $\underline{a}_{0}+T_{G^{\prime}}(\mathbb{C})$ and $\underline{a}_{1}+T_{G^{\prime}}(\mathbb{C})$ are $\mathbb{C}$-linearly independent in $T_{G}(\mathbb{C}) / T_{G^{\prime}}(\mathbb{C})$. Then it suffices to prove that $\underline{\widetilde{a}}_{0}+T_{G^{\prime}}(\mathbb{C})$ and $\underline{\widetilde{a}}_{1}+T_{G^{\prime}}(\mathbb{C})$ are $\mathbb{C}$-linearly independent. The proof is by contradiction. Suppose that there exist complex numbers $e_{1}, e_{2}$, not both zero, such that $e_{1} \underline{\widetilde{a}}_{0}+e_{2} \underline{\widetilde{a}}_{1} \in T_{G^{\prime}}(\mathbb{C})$. This means that

$$
e_{1}\left(\widetilde{a}_{00}, \widetilde{a}_{10}, \ldots, \widetilde{a}_{\delta 0}\right)+e_{2}\left(\widetilde{a}_{01}, \widetilde{a}_{11}, \ldots, \widetilde{a}_{\delta 1}\right) \in T_{G^{\prime}}(\mathbb{C}),
$$

that is,

$$
\left(e_{1} \widetilde{a}_{00}+e_{2} \widetilde{a}_{01}, e_{1} \widetilde{a}_{10}+e_{2} \widetilde{a}_{11}, \ldots, e_{1} \widetilde{a}_{\delta 0}+e_{2} \widetilde{a}_{\delta 1}\right) \in T_{G^{\prime}}(\mathbb{C}) .
$$

In what follows, we denote by $c_{i}(\delta)(i=1,2,3)$ positive numbers depending only on $\delta$. First, we note that $\underline{a}_{0}+T_{G^{\prime}}(\mathbb{C})$ and $\underline{a}_{1}+T_{G^{\prime}}(\mathbb{C})$ being linearly independent implies $G^{\prime}=\{0\} \times G_{2}^{\prime}$. Hence we have $T_{G^{\prime}}(\mathbb{C})=\{0\} \oplus T_{G_{2}^{\prime}}(\mathbb{C})$.

CASE 1: $\delta_{2}=\operatorname{dim} E^{\delta} / G_{2}^{\prime}=0$. This means $T_{G^{\prime}}(\mathbb{C})=\mathbb{C}^{\delta}$. Taking $f_{1}=0$ and $f_{2}=1$, we have

$$
f_{1} \underline{a}_{0}+f_{2} \underline{a}_{1} \in T_{G^{\prime}}(\mathbb{C}) .
$$


CASE 2: $\delta_{2}=\operatorname{dim} E^{\delta} / G_{2}^{\prime}>0$. From (20), we have $\left(e_{1} \widetilde{a}_{10}+e_{2} \widetilde{a}_{11}, \ldots\right.$ $\left.\ldots, e_{1} \widetilde{a}_{\delta 0}+e_{2} \widetilde{a}_{\delta 1}\right) \in T_{G_{2}^{\prime}}$. Then by the effective Kolchin theorem [10], there exist $\delta_{2}$ linearly independent integer points $\underline{\lambda}^{(1)}, \ldots, \underline{\lambda}^{\left(\delta_{2}\right)} \in \mathbb{Z}^{\delta}$ such that for any $\varrho$ with $1 \leq \varrho \leq \delta_{2}$,

$$
\begin{gathered}
\lambda_{1}^{(\varrho)}\left(e_{1} \widetilde{a}_{10}+e_{2} \widetilde{a}_{11}\right)+\cdots+\lambda_{\delta}^{(\varrho)}\left(e_{1} \widetilde{a}_{\delta 0}+e_{2} \widetilde{a}_{\delta 1}\right)=m_{1}^{(\varrho)} \omega_{1}+m_{2}^{(\varrho)} \omega_{2}, \\
\left\|\underline{\lambda}^{(\varrho)}\right\| \leq 2^{c_{1}(\delta)} D_{2}^{2 \delta_{2} /\left(\delta_{2}+1-\varrho\right)},
\end{gathered}
$$

where $\underline{\lambda}^{(\varrho)}=\left(\lambda_{1}^{(\varrho)}, \ldots, \lambda_{\delta}^{(\varrho)}\right)$. Thus from (20) we have the system of equations

$$
\begin{aligned}
& e_{1} \widetilde{a}_{00}+e_{2} \widetilde{a}_{01}=0, \\
& e_{1} \widetilde{p}_{10}+e_{2} \widetilde{p}_{11}=\xi_{1}, \\
& \ldots \ldots \ldots \ldots \ldots \ldots \ldots \\
& e_{1} \widetilde{p}_{\delta_{2} 0}+e_{2} \widetilde{p}_{\delta_{2} 1}=\xi_{\delta_{2}},
\end{aligned}
$$

where for brevity we put $\widetilde{p}_{\varrho 0}=\lambda_{1}^{(\varrho)} \widetilde{a}_{10}+\cdots+\lambda_{\delta}^{(\varrho)} \widetilde{a}_{\delta 0}, \widetilde{p}_{\varrho 1}=\lambda_{1}^{(\varrho)} \widetilde{a}_{11}+$ $\cdots+\lambda_{\delta}^{(\varrho)} \widetilde{a}_{\delta 1}$, and $\xi_{\varrho}=m_{1}^{(\varrho)} \omega_{1}+m_{2}^{(\varrho)} \omega_{2}\left(1 \leq \varrho \leq \delta_{2}\right)$. For any $i_{1}, i_{2}$ with $1 \leq i_{1}<i_{2} \leq \delta_{2}$, we easily obtain

$$
\begin{aligned}
& \left|p_{i_{1} 0}\right|,\left|p_{i_{1} 1}\right|,\left|\widetilde{p}_{i_{1} 0}\right|,\left|\widetilde{p}_{i_{1} 1}\right| \leq c_{38}\left\|\underline{\lambda}^{\left(i_{1}\right)}\right\|, \\
& \left|p_{i_{2} 0}\right|,\left|p_{i_{2} 1}\right|,\left|\widetilde{p}_{i_{2} 0}\right|,\left|\widetilde{p}_{i_{2} 1}\right| \leq c_{38}\left\|\underline{\lambda}^{\left(i_{2}\right)}\right\|, \\
& \left|\xi_{i_{1}}\right| \leq c_{39}\left\|\underline{\lambda}^{\left(i_{1}\right)}\right\|, \quad\left|\xi_{i_{2}}\right| \leq c_{39}\left\|\underline{\lambda}^{\left(i_{2}\right)}\right\| .
\end{aligned}
$$

Denote by $p_{\varrho s}$ the linear forms $\widetilde{p}_{\varrho s}$ of $\widetilde{a}_{h s}$ replaced by $a_{h s}\left(1 \leq \varrho \leq \delta_{2}, 1 \leq\right.$ $h \leq \delta, 0 \leq s \leq 1)$. Now we consider the system of linear equations analogous to $(21)$,

$$
\begin{aligned}
& a_{00} z_{1}+a_{01} z_{2}=0, \\
& p_{10} z_{1}+p_{11} z_{2}=\xi_{1}, \\
& \ldots \ldots \ldots \ldots \ldots \ldots \ldots \\
& p_{\delta_{2} 0} z_{1}+p_{\delta_{2} 1} z_{2}=\xi_{\delta_{2}} .
\end{aligned}
$$

Put

$$
P=\left(\begin{array}{cc}
a_{00} & a_{01} \\
p_{10} & p_{11} \\
\vdots & \vdots \\
p_{\delta_{2} 0} & p_{\delta_{2}}
\end{array}\right)
$$

Since $a_{00}=1, a_{01}=0, p_{10}=0$, and $p_{11}=\lambda_{1}^{(1)} \cdot 1+\lambda_{2}^{(1)} \beta+\cdots+\lambda_{\delta}^{(1)} \beta^{\delta-1} \neq 0$, we have $\operatorname{rank} P=2$. 
First, we assume $\left(\xi_{1}, \ldots, \xi_{\delta_{2}}\right) \neq(0, \ldots, 0)$. Then we shall show that

$$
\operatorname{rank}\left(\begin{array}{ccc}
a_{00} & a_{01} & 0 \\
p_{10} & p_{11} & \xi_{1} \\
\vdots & \vdots & \vdots \\
p_{\delta_{2} 0} & p_{\delta_{2} 1} & \xi_{\delta_{2}}
\end{array}\right)=2,
$$

which means that (23) has a nontrivial solution.

SubCASE 2.1: $\delta_{2}=1$. Then (23) has nontrivial solution (for example, take $\left.z_{1}=0, z_{2}=p_{11}^{-1} \xi_{1}\right)$.

SubCASE 2.2: $\delta_{2}>1$. From our assumption, (21) has a nontrivial solution $\left(e_{1}, e_{2}\right) \neq(0,0)$. Thus we have $\operatorname{rank} \widetilde{P}=\operatorname{rank}\left(\widetilde{P},{ }^{t} \xi\right)$, where $\xi=$ $\left(0, \xi_{1}, \ldots, \xi_{\delta_{2}}\right)$. Hence for any $i_{1}, i_{2}$ with $1 \leq i_{1}<i_{2} \leq \delta_{2}$, we have

$$
\left|\begin{array}{ccc}
\widetilde{a}_{00} & \widetilde{a}_{01} & 0 \\
\widetilde{p}_{i_{1} 0} & \widetilde{p}_{i_{1} 1} & \xi_{i_{1}} \\
\widetilde{p}_{i_{2} 0} & \widetilde{p}_{i_{2} 1} & \xi_{i_{2}}
\end{array}\right|=0 .
$$

By similar arguments to those in the exponential case, from (22) we have

$$
\operatorname{abs}\left(\left|\begin{array}{ccc}
a_{00} & a_{01} & 0 \\
p_{i_{1} 0} & p_{i_{1} 1} & \xi_{i_{1}} \\
p_{i_{2} 0} & p_{i_{2} 1} & \xi_{i_{2}}
\end{array}\right|\right) \leq \exp \left(-\frac{c}{4} \varrho(S)\right) .
$$

Next, we shall find a lower bound of the absolute value of the determinant

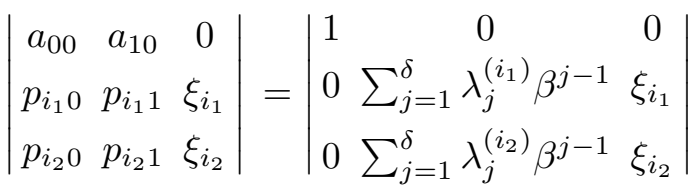

$$
\begin{aligned}
& =\left|\begin{array}{cc}
\sum_{j=1}^{\delta} \lambda_{j}^{\left(i_{1}\right)} \beta^{j-1} & m_{1}^{\left(i_{1}\right)} \\
\sum_{j=1}^{\delta} \lambda_{j}^{\left(i_{2}\right)} \beta^{j-1} & m_{1}^{\left(i_{2}\right)}
\end{array}\right| \omega_{1}+\left|\begin{array}{cc}
\sum_{j=1}^{\delta} \lambda_{j}^{\left(i_{1}\right)} \beta^{j-1} & m_{2}^{\left(i_{1}\right)} \\
\sum_{j=1}^{\delta} \lambda_{j}^{\left(i_{2}\right)} \beta^{j-1} & m_{2}^{\left(i_{2}\right)}
\end{array}\right| \omega_{2} \\
& =: \zeta_{1} \omega_{1}+\zeta_{2} \omega_{2} \text {. }
\end{aligned}
$$

It is clear that $\zeta_{1}, \zeta_{2} \in \mathbb{Q}(\beta)$ and $\operatorname{deg} \zeta_{1}, \operatorname{deg} \zeta_{2} \leq \delta$.

By an elementary computation, we see easily that

$$
H\left(\zeta_{1}\right), H\left(\zeta_{2}\right) \leq\left(c_{40}\left\|\underline{\lambda}^{\left(i_{1}\right)}\right\|\left\|\underline{\lambda}^{\left(i_{2}\right)}\right\|\right)^{c_{1}(\delta)} \leq\left(c_{41} D_{2}^{3 \delta_{2}}\right)^{c_{1}(\delta)} \leq c_{42} D_{2}^{c_{2}(\delta)},
$$

where $H(\zeta)$ denotes the usual height of an algebraic number $\zeta$. If $\zeta_{1} \omega_{1}+$ $\zeta_{2} \omega_{2} \neq 0$, we have

$$
\left|\zeta_{1} \omega_{1}+\zeta_{2} \omega_{2}\right| \geq C \exp \left(-c_{3}(\delta)\left(\log D_{2}\right)^{\tau_{0}}\right),
$$

where $\tau_{0}>1$ is some absolute constant and $C>0$ is a number depending 
only on $\omega_{1}, \omega_{2}$ and $\delta$ (see [9, Theorem 1]). Thus in this case, we have

$$
\operatorname{abs}\left(\left|\begin{array}{ccc}
a_{00} & a_{10} & 0 \\
p_{i_{1} 0} & p_{i_{1} 1} & \xi_{i_{1}} \\
p_{i_{2} 0} & p_{i_{2} 1} & \xi_{i_{2}}
\end{array}\right|\right) \geq C \exp \left(-c_{3}(\delta)\left(\log D_{2}\right)^{\tau_{0}}\right)
$$

which contradicts (24), since $S$ is sufficiently large. Thus we have

$$
\left|\begin{array}{ccc}
a_{00} & a_{10} & 0 \\
p_{i_{1} 0} & p_{i_{1} 1} & \xi_{i_{1}} \\
p_{i_{2} 0} & p_{i_{2} 1} & \xi_{i_{2}}
\end{array}\right|=0 \quad\left(1 \leq i_{1}<i_{2} \leq \delta_{2}\right) .
$$

This means $\operatorname{rank} P=\operatorname{rank}\left(P,{ }^{t} \underline{\xi}\right)=2$, and hence (23) has a nontrivial solution.

In the case of $\left(\xi_{1}, \ldots, \xi_{\delta_{2}}\right)=(0, \ldots, 0)$, we have $\operatorname{rank} \widetilde{P}<2$; and we can also show that $\operatorname{rank} P<2$, which will be excluded.

Thus (23) always has a nontrivial solution $\left(f_{1}, f_{2}\right) \neq(0,0)$ (say). Now by similar arguments to those in the exponential case, we have $f_{1} \underline{a}_{0}+f_{2} \underline{a}_{1} \in$ $T_{G^{\prime}}(\mathbb{C})$, which is a contradiction.

This completes the proof of Lemma 7.

End of proof of Corollary 7. Since hypotheses $\left(\mathrm{H}_{\mathrm{A}}\right)$ and $\left(\mathrm{H}_{\mathrm{B}}\right)$ are satisfied, we can now apply Corollary 6 . We put $\underline{\omega}^{(4)}=\left(1,0, \ldots, 0,0, \beta^{j-1}, \beta^{j-1}\right.$, $\left.\wp\left(\beta^{s} u\right) ; 1 \leq j \leq \delta, 0 \leq s \leq(\delta-1)^{2}\right), \underline{\omega}^{\prime}=\left(\wp\left(\beta^{s} u\right) ; 0 \leq s \leq \delta-1\right)$. Then it is clear that $\operatorname{deg} \operatorname{tr}_{\mathbb{Q}} \mathbb{Q}\left(\underline{\omega}^{(4)}\right)=\operatorname{deg} \operatorname{tr}_{\mathbb{Q}} \mathbb{Q}\left(\underline{\omega}^{\prime}\right)$.

If $\wp$ has no complex multiplications, we have $\mathbb{F}=\mathbb{Q}$, and hence $\kappa=$ $(\delta+2) / 3$. Thus if $\delta \geq 2$, we have $\kappa>4 / 3$, and we deduce from Corollary 6 that $\operatorname{deg} \operatorname{tr}_{\mathbb{Q}} \mathbb{Q}\left(\underline{\omega}^{(4)}\right) \geq[(\delta+2) / 3]$.

If $\wp$ has complex multiplications, we have $[\mathbb{F}: \mathbb{Q}]=2$, and hence $\kappa=$ $(\delta+1) / 2$. If $\delta \geq 2$, we have $\kappa \geq 3 / 2$, and we infer from Corollary 6 that $\operatorname{deg} \operatorname{tr}_{\mathbb{Q}} \mathbb{Q}\left(\underline{\omega}^{(4)}\right) \geq[(\delta+1) / 2]$.

Acknowledgements. I am grateful to the referee for his valuable suggestions and useful comments.

\section{References}

[1] M. Ably, Résultats quantitatifs d'indépendance algébrique pour les groupes algébriques, J. Number Theory 42 (1992), 194-231.

[2] A. Baker, Transcendental Number Theory, Cambridge Univ. Press, Cambridge, 1975.

[3] D. Brownawell, Some remarks on semi-resultants, in: Transcendence Theory: Advances and Applications, A. Baker and D. W. Masser (eds.), Academic Press, London, 1977, 205-210.

[4] G.-L. Chen, Application de la méthode de Baker à de grands degrés de transcendance, C. R. Acad. Sci. Paris 320 (1995), 523-528. 
[5] G. V. Chudnovsky, Contribution to the Theory of Transcendental Numbers, Amer. Math. Soc. Surveys and Monographs 19, Providence, 1984.

[6] G. Diaz, Lemme de zéros pour des polynômes exponentiels, Sém. Théorie des Nombres, Saint-Etienne, 1987-1988.

[7] —, Grands degrés de transcendance pour des familles d'exponentielles, J. Number Theory 31 (1989), 1-23.

[8] —, Degré de transcendance 2 pour familles d'exponentielles par la méthode de Baker, Sém. d'Arithmétique, Saint-Etienne, 1990-91-92.

[9] D. Masser, Elliptic Functions and Transcendence, Lecture Notes in Math. 437, Springer, 1975.

[10] D. Masser and G. Wüstholz, Fields of large transcendence degree generated by values of elliptic functions, Invent. Math. 72 (1983), 407-464.

[11] P. Philippon, Variétés abéliennes et indépendance algébrique II: Un analogue du théorème de Lindemann-Weierstrass, ibid., 389-405.

[12] —, Critères pour l'indépendance algébrique, Publ. I.H.E.S. 64 (1986), 5-52.

[13] —, Lemmes de zéros dans les groupes algébriques commutatifs, Bull. Soc. Math. France 114 (1986), 355-383.

[14] P. Philippon et M. Waldschmidt, Formes linéaires de logarithmes sur les groupes algébriques commutatifs, Illinois J. Math. 32 (1988), 281-314.

[15] E. Reyssat, Approximation algébrique de nombres liés aux fonctions elliptiques, Bull. Soc. Math. France 108 (1980), 47-79.

[16] J.-P. Serre, Quelques propriétés des groupes algébriques commutatifs, appendix to [19], 191-202.

[17] R. Tubbs, Algebraic groups and small transcendence degree I, J. Number Theory 25 (1987), 279-307.

[18] M. Waldschmidt, Utilisation de la méthode de Baker dans des problèmes d'indépendance algébrique, C. R. Acad. Sci. Paris 275 (1972), 1215-1217.

[19] —, Nombres transcendants et groupes algébriques, Astérisque 69-70 (1979).

[20] —, Sous-groupes analytiques de groupes algébriques, Ann. of Math. 117 (1983), 627-657.

[21] —, Groupes algébriques et grands degrés de transcendance, Acta Math. 156 (1986), 253-302.

[22] - Lemmes de zéros pour les polynômes exponentiels, Sém. Théorie des Nombres, Saint-Etienne, 1986-1987.

[23] - On the transcendence methods of Gelfond and Schneider in several variables, in: New Advances in Transcendence Theory, A. Baker (ed.), Cambridge Univ. Press, Cambridge, 1988, 375-398.

Department of Mathematical Sciences

Ibaraki University

Mito, Ibaraki 310-8512, Japan

E-mail: mtake@mx.ibaraki.ac.jp

Received on 28.5.2001

and in revised form on 15.6.2005 